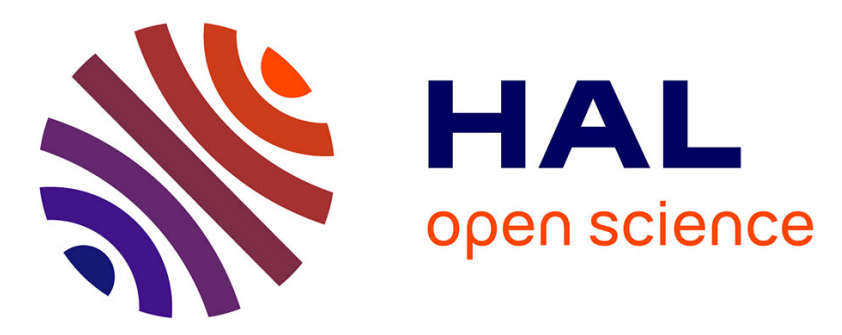

\title{
Eternal Domination: D-Dimensional Cartesian and Strong Grids and Everything in Between
}

Fionn Mc Inerney, Nicolas Nisse, Stéphane Pérennes

\section{To cite this version:}

Fionn Mc Inerney, Nicolas Nisse, Stéphane Pérennes. Eternal Domination: D-Dimensional Cartesian and Strong Grids and Everything in Between. Algorithmica, 2021, 83 (5), pp.1459-1492. 10.1007/s00453-020-00790-8 . hal-02801932v2

\section{HAL Id: hal-02801932 \\ https://hal.inria.fr/hal-02801932v2}

Submitted on 26 May 2021 (v2), last revised 21 Feb 2022 (v3)

HAL is a multi-disciplinary open access archive for the deposit and dissemination of scientific research documents, whether they are published or not. The documents may come from teaching and research institutions in France or abroad, or from public or private research centers.
L'archive ouverte pluridisciplinaire HAL, est destinée au dépôt et à la diffusion de documents scientifiques de niveau recherche, publiés ou non, émanant des établissements d'enseignement et de recherche français ou étrangers, des laboratoires publics ou privés. 


\title{
Eternal Domination: D-Dimensional Cartesian and Strong Grids and Everything in Between*
}

\author{
Fionn Mc Inerney ${ }^{\dagger 1,2}$, Nicolas Nisse $^{2}$, and Stéphane Pérennes ${ }^{2}$ \\ ${ }^{1}$ Laboratoire d'Informatique et Systèmes, Aix-Marseille Université, CNRS, and Université de Toulon \\ Faculté des Sciences de Luminy, Marseille, France \\ ${ }^{2}$ Université Côte d'Azur, Inria, CNRS, I3S, France
}

\begin{abstract}
In the eternal domination game played on graphs, an attacker attacks a vertex at each turn and a team of guards must move a guard to the attacked vertex to defend it. The guards may only move to adjacent vertices on their turn. The goal is to determine the eternal domination number $\gamma_{\text {all }}^{\infty}$ of a graph, which is the minimum number of guards required to defend against an infinite sequence of attacks. This paper first continues the study of the eternal domination game on strong grids $P_{n} \otimes P_{m}$. Cartesian grids $P_{n} \square P_{m}$ have been vastly studied with tight bounds existing for small grids such as $k \times n$ grids for $k \in\{2,3,4,5\}$. It was recently proven that $\gamma_{\text {all }}^{\infty}\left(P_{n} \square P_{m}\right)=\gamma\left(P_{n} \square P_{m}\right)+O(n+m)$ where $\gamma\left(P_{n} \square P_{m}\right)$ is the domination number of $P_{n} \square P_{m}$ which lower bounds the eternal domination number [Lamprou et al. Eternally dominating large grids. Theoretical Computer Science, 794:27-46, 2019]. We prove that, for all $n, m \in \mathbb{N}^{*}$ such that $m \geq n,\left\lfloor\frac{n}{3}\right\rfloor\left\lfloor\frac{m}{3}\right\rfloor+\Omega(n+m)=\gamma_{\text {all }}^{\infty}\left(P_{n} \otimes P_{m}\right)=\left\lceil\frac{n}{3}\right\rceil\left\lceil\frac{m}{3}\right\rceil+O(m \sqrt{n})$ (note that $\left\lceil\frac{n}{3}\right\rceil\left\lceil\frac{m}{3}\right\rceil$ is the domination number of $\left.P_{n} \otimes P_{m}\right)$. We then generalise our technique to prove that $\gamma_{\text {all }}^{\infty}(G)=\gamma(G)+o(\gamma(G))$ for all graphs $G \in \mathcal{F}$, where $\mathcal{F}$ is a large family of $D$-dimensional grids which are supergraphs of the $D$-dimensional Cartesian grid and subgraphs of the $D$ dimensional strong grid. In particular, $\mathcal{F}$ includes both the $D$-dimensional Cartesian grid and the $D$-dimensional strong grid.
\end{abstract}

Keywords: Eternal Domination, Games, Graphs, Grids, Cartesian grid, Strong grid, King's graph

\section{Introduction}

The origins of the eternal domination game date back to the 1990's where the military strategy of Emperor Constantine for defending the Roman Empire was studied in a mathematical setting [1, $22,23,25]$. Roughly, a limited number of armies must be placed in such a way that an army can always move to defend against an attack by invaders.

Precisely, eternal domination is a 2-player game on graphs introduced in [6] and defined as follows. Initially, $k$ guards are placed on some vertices of a graph $G=(V, E)$. Turn-by-turn, an

${ }^{*}$ This work has been partially supported by the ANR project DISTANCIA (ANR-14-CE25-0006). An extended abstract of part of this paper has been presented in [15].

${ }^{\dagger}$ Corresponding author. Email address: fmcinern@gmail.com 
attacker first chooses a vertex $v \in V$ to attack. Then, if no guard is occupying $v$ or a vertex adjacent to $v$, then the attacker wins. Otherwise, one guard must move along an edge to occupy $v$ if it is not already occupied, and the next turn starts. If the attacker never wins whatever be its sequence of attacks, then the guards win. So, clearly, there is no point in the attacker attacking an occupied vertex. The aim in eternal domination is to minimise the number of guards that must be used in order to win. Hence, let $\gamma^{\infty}(G)$ be the minimum integer $k$ such that there exists a strategy allowing $k$ guards to win, regardless of what the attacker does [6].

In this paper, we consider the "all guards move" variant of eternal domination, proposed in [12], where, at their turn, every guard may move to a neighbour of its position (still satisfying that the attacked vertex is occupied by a guard at the end of the turn). Let $\gamma_{\text {all }}^{\infty}(G)$ be the minimum number of guards for which a winning strategy exists in this setting. By definition, $\gamma(G) \leq \gamma_{\text {all }}^{\infty}(G) \leq \gamma^{\infty}(G)$ for any graph $G$ where $\gamma(G)$ is the minimum size of a dominating set in $G^{1}$.

Variants of the eternal domination game also differ in the fact that one or more guards may simultaneously occupy the same vertex. In the initial variant where a single guard is allowed to move each turn, this is not a strong constraint [6]. That is, imposing that a vertex cannot be occupied by more than one guard does not increase the number of guards required to win. In the case when multiple guards may move each turn, there are some graphs where this constraint increases the number of guards [19]. Let $\gamma_{a l l}^{* \infty}(G)$ be the minimum number of guards to win in $G$, moving several guards per turn, and in such a way that a vertex cannot be occupied by several guards.

Previous works mainly studied lower and upper bounds for $\gamma^{\infty}(G)$ and $\gamma_{\text {all }}^{\infty}(G)$ in function of other parameters of $G$, such as its domination number $\gamma(G)[12]$, independence number $\alpha(G)^{2}[6,12]$, and clique cover number $\theta(G)^{3}[6]$. Notably, these results give the following chain of inequalities: $\gamma(G) \leq \gamma_{\text {all }}^{\infty}(G) \leq \alpha(G) \leq \gamma^{\infty}(G) \leq \theta(G)[6]$. Particular graph classes have also been studied such as paths and cycles [12], trees [17], and interval graphs [24]. In particular, the class of grids and graph products has been widely studied $[4,10,11,13,19,20,21,26]$.

In this paper, we first focus on the class of strong grids $S G$ and provide an almost tight asymptotical value for $\gamma_{\text {all }}^{\infty}(S G)$. Our result also holds for $\gamma_{\text {all }}^{* \infty}(S G)$. Our main result is a generalisation of this new technique (used for strong grids) to prove upper bounds for a large family of higher dimensional grids. In particular, we prove that $\gamma_{\text {all }}^{\infty}(G)=\gamma(G)+o(\gamma(G))$ for all graphs $G \in \mathcal{F}$, where $\mathcal{F}$ is a family of $D$-dimensional grids which are supergraphs of the $D$-dimensional Cartesian grid and subgraphs of the $D$-dimensional strong grid.

\subsection{Related Work}

In terms of complexity, the "all guards move" variant of eternal domination was shown to be NPcomplete in Hamiltonian split graphs [3]. Note that it is not known whether the decision problem associated to $\gamma_{\text {all }}^{\infty}$ is in NP in general graphs. Moreover, given a graph $G$ and an integer $k$ as inputs, the problem of deciding if $\gamma^{\infty}(G) \leq k$ is coNP-hard [2].

Several graph classes have also been studied. For a path $P_{n}$ on $n$ vertices, $\gamma_{\text {all }}^{\infty}\left(P_{n}\right)=\left\lceil\frac{n}{2}\right\rceil$ and for a cycle $C_{n}$ on $n$ vertices, $\gamma_{\text {all }}^{\infty}\left(C_{n}\right)=\left\lceil\frac{n}{3}\right\rceil$ [12]. In [17], the authors present a linear-time algorithm to determine $\gamma_{\text {all }}^{\infty}(T)$ for all trees $T$. It was proven that $\gamma_{\text {all }}^{\infty}(G)=\alpha(G)$ for all interval graphs $G$ [24]. In the past few years, a lot of effort was put in by several authors to determine

\footnotetext{
${ }^{1} D \subseteq V$ is a dominating set of $G$ if every vertex is in $D$ or adjacent to a vertex in $D$.

${ }^{2} \alpha(\bar{G})$ is the maximum size of an independent set in $G$.

${ }^{3} \theta(G)$ is the minimum number of complete subgraphs of $G$ whose union covers $V(G)$.
} 
the eternal domination number of Cartesian grids, $\gamma_{a l l}^{\infty}\left(P_{n} \square P_{m}\right)$. Exact values were determined for $2 \times n$ Cartesian grids [13] and $4 \times n$ Cartesian grids [4]. Asymptotically tight bounds for $3 \times n$ Cartesian grids were obtained in [10] and improved in [21]. Finally, bounds for $5 \times n$ Cartesian grids were given in [26]. The best known lower bound for $\gamma_{\text {all }}^{\infty}\left(P_{n} \square P_{m}\right)$ for values of $n$ and $m$ large enough, is the domination number with the latter only being recently determined in [14]. The best known upper bound for $\gamma_{\text {all }}^{\infty}\left(P_{n} \square P_{m}\right)$ was determined recently in [20], where it was shown that $\gamma_{\text {all }}^{\infty}\left(P_{n} \square P_{m}\right)=\gamma\left(P_{n} \square P_{m}\right)+O(n+m)$. Using similar techniques to those used in [20], Gagnon et al. showed that $\gamma_{\text {all }}^{\infty}\left(P_{n} \otimes P_{m}\right) \leq \frac{m n}{7}+O(m+n)$ in [11], a result which we improve upon in Theorem 8. Note that all the results discussed in this subsection also hold for $\gamma_{\text {all }}^{* \infty}$.

There are also many other variants of the game that exist and here we give a brief description and references for some of them. Recently, the eternal domination game and a variant have been studied in digraphs, including orientations of grids and toroidal strong grids [2]. Eternal total domination was studied in [18], where a total dominating set must be maintained by the guards each turn. The eviction model of eternal domination was studied in [16], where a vertex containing a guard is attacked each turn, which forces the guard to move to an adjacent empty vertex with the condition that the guards must maintain a dominating set each turn. The authors of the current paper studied a generalisation of eternal domination, called the Spy game, in [7, 8]. For more information and results on the original eternal domination game and its variants, see the survey [19].

\subsection{Our results}

The first result of this paper is that, for all $n, m \in \mathbb{N}^{*}$ such that $m \geq n$,

$$
\left\lfloor\frac{n}{3}\right\rfloor\left\lfloor\frac{m}{3}\right\rfloor+\Omega(n+m)=\gamma_{\text {all }}^{\infty}\left(P_{n} \otimes P_{m}\right)=\left\lceil\frac{n}{3}\right\rceil\left\lceil\frac{m}{3}\right\rceil+O(m \sqrt{n}) .
$$

We prove that this result also holds in the case when at most one guard may occupy each vertex (see Section 5).

Note that, in toroidal strong grids $C_{n} \otimes C_{m}$, the problem is trivial and $\gamma_{\text {all }}^{\infty}\left(C_{n} \otimes C_{m}\right)=\left\lceil\frac{n}{3}\right\rceil\left\lceil\frac{m}{3}\right\rceil$ for any $n$ and $m$. However, in strong grids, border-effects make the problem much harder. The upper bound is proven by defining a set of specific configurations that each dominate the grid and are "invariant" to the movements required by the defined strategy to defend against attacks. That is, the attacks are separated into three types of attacks: horizontal, vertical, and diagonal, and the strategy defined gives the movement of the guards based on the type of attack. It is shown that in each of the three cases of attacks, the guards are able to move from their current configuration to another configuration in the set of configurations (so, it does not matter which configuration was the initial one and which new configuration the guards reach after their moves) and hence, the guards can defend against an infinite sequence of attacks.

The lower bound is proven by showing that, in any winning configuration in eternal domination, there are some vertices that are dominated by more than one guard, and/or some guards dominate at most 6 vertices. By double counting, this leads to the necessity of having $\Omega(n+m)$ extra guards compared to the classical domination $($ when $n \equiv 0(\bmod 3)$ and $m \equiv 0(\bmod 3))$.

The main result of this paper, which is a generalisation of the technique used for obtaining the upper bound for strong grids, is then presented. That is, it is proven that $\gamma_{\text {all }}^{\infty}(G)=\gamma(G)+o(\gamma(G))$ for all graphs $G \in \mathcal{F}$, where $\mathcal{F}$ is a family of graphs which are supergraphs of $P_{n_{1}} \square \cdots \square P_{n_{D}}$ and subgraphs of $P_{n_{1}} \otimes \cdots \otimes P_{n_{D}}$. In particular, $\mathcal{F}$ includes both the $D$-dimensional Cartesian grid and the $D$-dimensional strong grid. 


\section{Preliminaries}

We use classic graph-theory terminology [9]. Notably, given a graph $G=(V, E)$ and $S \subseteq V$, let $N(S)=\{v \in V \backslash S \mid \exists w \in S,\{v, w\} \in E\}$ denote the set of neighbours (not in $S$ ) of the vertices in $S$ and let $N[S]=N(S) \cup S$ denote the closed neighbourhood of $S$. For $v \in V$, let $N(v)=N(\{v\})$ and $N[v]=N(v) \cup\{v\}$.

Let $n, m \in \mathbb{N}^{*}$ be such that $m \geq n$ and let the $n \times m$ strong grid, denoted by $S G_{n \times m}$, be the strong product $P_{n} \otimes P_{m}$ of an $n$-node path with an $m$-node path. Precisely, $S G_{n \times m}$ is the graph with the set of vertices $\{(i, j) \mid 1 \leq i \leq n, 1 \leq j \leq m\}$, and two vertices $\left(i_{1}, j_{1}\right)$ and $\left(i_{2}, j_{2}\right)$ are adjacent if and only if $\max \left\{\left|i_{2}-i_{1}\right|,\left|j_{2}-j_{1}\right|\right\}=1$. That is, the vertices are identified by their Cartesian coordinates, i.e., the vertex $(i, j)$ is the vertex in row $i$ and column $j$. The vertex $(1,1)$ is in the bottom-left corner and the vertex $(n, m)$ is in the top-right corner.

Definition 1. The set of border vertices of $S G_{n \times m}$ is the set

$$
B=\bigcup_{1 \leq i \leq n, 1 \leq j \leq m}\{(1, j),(n, j),(i, 1),(i, m)\} \text { of vertices of degree } \leq 5 .
$$

The set of pre-border vertices of $S G_{n \times m}$ is the set $P B=N(B)$.

Equivalently, $P B$ is the set of border vertices of the strong grid induced by $V\left(S G_{n \times m}\right) \backslash B$.

We consider the turn-by-turn 2-player game in graphs called eternal domination. Each turn, each vertex of a graph $G=(V, E)$ may be occupied by one or more guards. Let $k \in \mathbb{N}^{*}$ be the total number of guards. The positions of the guards are formally defined by a multi-set $C$ of vertices, called a configuration, where the number of occurrences of a vertex $v \in C$ corresponds to the number of guards at $v \in V$ and $k=|C|$. Each turn, given a current configuration $C=\left\{v_{i} \mid 1 \leq i \leq k\right\}$ of $k$ guards, Player 1 , the attacker, attacks a vertex $v \in V$. Then, Player 2 (the defender) may move each of its guards to a neighbour of their current position, thereby, achieving a new configuration $C^{\prime}=\left\{w_{i} \mid 1 \leq i \leq k\right\}$ such that $w_{i} \in N\left[v_{i}\right]$ for every $1 \leq i \leq k$ (we then say that $C^{\prime}$ is compatible with $C$, which is clearly a symmetric relation). If $v \notin C^{\prime}$, then the attacker wins, otherwise, the game goes on with a next turn (given the new configuration $C^{\prime}$ ).

A strategy for $k$ guards is defined by an initial configuration of size $k$ and by a function that, for every current configuration $C$ and every attacked vertex $v \in V$, specifies a new configuration $C^{\prime}$ compatible with $C$. A strategy $\mathcal{S}$ for the guards is winning if, for every sequence of attacked vertices, the attacker never wins when the defender plays according to $\mathcal{S}$.

Our first main contribution is the design of a winning strategy for $\gamma\left(S G_{n \times m}\right)+o\left(\gamma\left(S G_{n \times m}\right)\right)$ guards in $S G_{n \times m}$, where $\gamma\left(S G_{n \times m}\right)=\left\lceil\frac{n}{3}\right\rceil\left\lceil\frac{m}{3}\right\rceil$ is the domination number of $S G_{n \times m}$. The next lemma is key for this winning strategy.

In our strategy, it will often be useful to move a guard from a node $u \in P B$ of the pre-border to another node $v \in P B$ such that $u$ and $v$ are not necessarily adjacent. For this purpose, the idea is to place a sufficient number of guards on the vertices of the border such that a "flow" of the guards on the border vertices will simulate the move of the guard from $u$ to $v$ in one turn.

Precisely, given a configuration $C$ and $u, v \in V\left(S G_{n \times m}\right)$ with $u \in C$, a guard is said to jump from $u$ to $v$ if the configuration $(C \backslash\{u\}) \cup\{v\}$ is compatible with $C$, i.e., the guards, in one turn, can move to achieve the same configuration as $C$ except that there is one less guard on $u$ and one more guard on $v$. More generally, given $U \subset C$ and $W \subset V\left(S G_{n \times m}\right)$, a set of guards is said to jump from $U$ to $W$ if the configuration $(C \backslash U) \cup W$ is compatible with the configuration $C$. 
Lemma 2. Let $\alpha, \beta \in \mathbb{N}^{*}$ such that $\beta \leq \alpha$. Let $U, W \subseteq P B$ be two subsets of pre-border vertices such that $|U|=|W|=\beta$. In any configuration $C$ such that $U \subseteq C$ and $C$ contains at least $\alpha$ occurrences of each vertex in $B$ (i.e., each border vertex is occupied by at least $\alpha$ guards), $\beta$ guards may "jump" from $U$ to $W$ in one turn. Moreover, only guards in $U \cup B$ move.

Proof. The proof is by induction on $\beta$. The inductive hypothesis is that if each vertex in $B$ contains $\alpha$ guards, then $\beta \leq \alpha$ guards may "jump" from $U$ to $W$ in one turn such that at most $\beta$ guards move off of each vertex $w \in B$ in this turn. For the base case, let us assume that $U=\{u\}$ and $W=\{w\}$. Let us show how 1 guard can "jump" from $u$ to $w$ in one turn. If $u=w$, the result trivially holds, so let $u \neq w$. Let $u^{\prime} \in B$ (resp., $w^{\prime}$ ) be a neighbour of $u$ (of $w$ ) that shares one coordinate with $u$ (with $w$ ). Let $Q=\left(u^{\prime}=v_{0}, v_{1}, \ldots, v_{\ell}=w^{\prime}\right)$ be a path from $u^{\prime}$ to $w^{\prime}$ induced by the border vertices. In one turn, a guard at $u$ moves to $u^{\prime}$, for every $0 \leq i<\ell$, a guard at $v_{i}$ moves to $v_{i+1}$, and a guard at $v_{\ell}$ moves to $w$.

Now, assume the inductive hypothesis holds for $\beta \geq 1$. If $\beta=\alpha$, we are done, so assume $\beta<\alpha$. Let $|U|=|W|=\beta+1 \leq \alpha$ and let $u \in U$ and $w \in W$. By the inductive hypothesis, $\beta$ guards may jump from $U \backslash\{u\}$ to $W \backslash\{w\}$ in one turn in such a way that, for every vertex $b \in B$, at most $\beta$ guards move off of $b$ during this turn. Since every vertex of $B$ is occupied by $\alpha>\beta$ guards, at least one guard is unused on every vertex of $B$. Thus, it possible to use the same strategy as in the base case to make one guard jump from $u$ to $w$ on this same turn.

\section{Upper bound strategy}

This section is devoted to proving that, for all $n, m \in \mathbb{N}^{*}$ such that $m \geq n, \gamma_{\text {all }}^{\infty}\left(S G_{n \times m}\right)=$ $\left\lceil\frac{n}{3}\right\rceil\left\lceil\frac{m}{3}\right\rceil+O(m \sqrt{n})$.

Before considering the general case, let us first assume that $n-2 \equiv 0(\bmod 3)$ and that there exists $k \in \mathbb{N}^{*}$ such that $k-2 \equiv 0(\bmod 3)$, and $m \equiv 0(\bmod k)$. The $n \times m$ strong grid will be partitioned into blocks which are subgrids of size $n \times k$. More precisely, for all $1 \leq q \leq \frac{m}{k}$, the $q^{\text {th }}$ block contains columns $(q-1) k+1$ through $q k$ of $S G_{n \times m}$.

\subsection{Horizontal attacks}

In this section, we only consider one block of $S G_{n \times m}$. W.l.o.g., let us consider the block $S G_{n \times k}$ induced by $\{(i, j) \mid 1 \leq i \leq n, 1 \leq j \leq k\}$. Let us first define a family of parameterised configurations for this block.

Let $\mathcal{X}=\left\{\left(b, a_{1}, \ldots, a_{\frac{n-2}{3}}\right) \mid b \in\{1,2,3\}, a_{i} \in\{1,2,3\}\right.$ for $\left.i=1, \ldots, \frac{n-2}{3}\right\}$.

Given $X=\left(b, a_{1}, \ldots, a_{\frac{n-2}{3}}\right) \in \mathcal{X}$, let $x_{i}(X)=3(i-1)+b+1$, and $y_{j, i}(X)=3(j-1)+a_{i}+1$

for every $1 \leq i \leq \frac{n-2}{3}$ and $1 \leq j \leq \frac{k-2}{3}$. We set $x_{i}=x_{i}(X)$ and $y_{j, i}=y_{j, i}(X)$ when there is no ambiguity. Intuitively, $b$ will represent the vertical shift of the positions of the guards in configuration $X$. Similarly, for every $1 \leq i \leq \frac{n-2}{3}, a_{i}$ represents the horizontal shift of the positions of the guards in row $x_{i}(X)$ in configuration $X$ (see Figure 1).

Horizontal Configurations. Let us define the set $\mathcal{C}_{H}$ of configurations as follows. For every $X \in \mathcal{X}$, let $C_{H}(X)=B \cup\left\{\left(x_{i}(X), y_{j, i}(X)\right) \mid 1 \leq i \leq \frac{n-2}{3}, 1 \leq j \leq \frac{k-2}{3}\right\}$ be the configuration where there is one guard at every vertex of $B$ and one guard at each vertex $\left(x_{i}(X), y_{j, i}(X)\right)=$ $\left(3(i-1)+b+1,3(j-1)+a_{i}+1\right)$ for every $1 \leq i \leq \frac{n-2}{3}$ and $1 \leq j \leq \frac{k-2}{3}$. See an example in 
Figure 1. Then,

$$
\mathcal{C}_{H}=\left\{C_{H}(X) \mid X \in \mathcal{X}\right\}
$$

Note that $\left|C_{H}(X)\right|=\frac{(n-2)(k-2)}{9}+2(n+k)-4=\kappa_{H}$ for every $X \in \mathcal{X}$. That is, any horizontal configuration uses $\kappa_{H}$ guards.

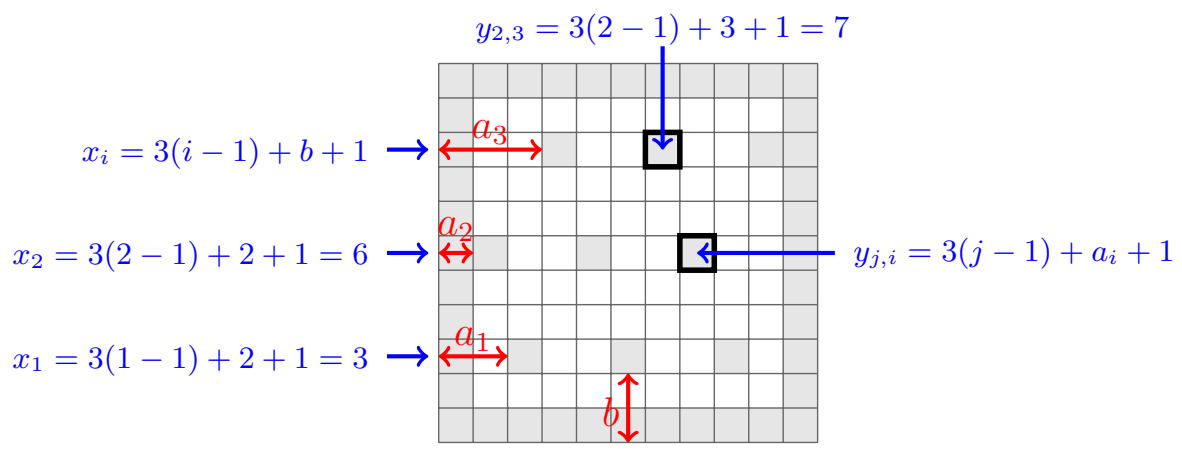

Figure 1: $P_{11} \otimes P_{11}$ where the squares are vertices and two squares sharing a side and/or a corner are adjacent. Example of a configuration $C_{H}(X)$ where $X=(b=$ $\left.2, a_{1}=2, a_{2}=1, a_{3}=a_{\frac{n-2}{3}}=3\right)$, there is one guard at each square in gray, and the white squares contain no guards.

Lemma 3. Every configuration $C_{H}(X) \in \mathcal{C}_{H}$ is a dominating set of the block $S G_{n \times k}$.

Proof. The pre-border and border vertices are dominated by the guards on the border vertices. For all $i, j \in \mathbb{N}^{*}$ such that $1 \leq i \leq \frac{n-2}{3}$ and $1 \leq j \leq \frac{k-2}{3}$, the guards on the vertices $\left(x_{i}, y_{j, i}\right)$ dominate the vertices $\left\{\left(x_{i}+1, y_{j, i}\right),\left(x_{i}-1, y_{j, i}\right),\left(x_{i}, y_{j, i}-1\right),\left(x_{i}, y_{j, i}+1\right),\left(x_{i}+1, y_{j, i}+1\right),\left(x_{i}+1, y_{j, i}-1\right),\left(x_{i}-\right.\right.$ $\left.\left.1, y_{j, i}-1\right),\left(x_{i}-1, y_{j, i}+1\right)\right\}$.

In this subsection, we limit the power of the attacker by allowing it to attack only some predefined vertices (this kind of attack will be referred to as a horizontal attack). For every configuration $C_{H}(X) \in \mathcal{C}_{H}$ and for any such attack, we show that the guards may be moved (in one turn) in such a way to defend the attacked vertex and reach a new configuration in $\mathcal{C}_{H}$.

Horizontal Attacks. Let $X=\left(b, a_{1}, \ldots, a_{\frac{n-2}{3}}\right) \in \mathcal{X}$ and $C_{H}(X) \in \mathcal{C}_{H}$. Let

$$
A_{H}(X)=\left\{\left(x_{i}, y\right) \mid 1 \leq i \leq \frac{n-2}{3}, 1 \leq y \leq k\right\}
$$

A horizontal attack with respect to $X$ is an attack at any vertex in $A_{H}(X)$, i.e., an attack at any vertex of a row where some non-border vertex is occupied by a guard. Note that, for every vertex $v \in A_{H}(X)$, either $v$ is occupied by a guard or there is a guard on the vertex to the left or to the right of $v$. In Figure 2, red squares represent the vertices of $A_{H}(X) \backslash C_{H}(X)$.

The next lemma proves that, from any horizontal configuration and against any horizontal attack (with respect to this current configuration), there is a possible strategy for the guards that defends against this attack and leads to a (new) horizontal configuration. Therefore, starting from any horizontal configuration, there is a strategy of the guards that wins against any sequence of horizontal attacks. 


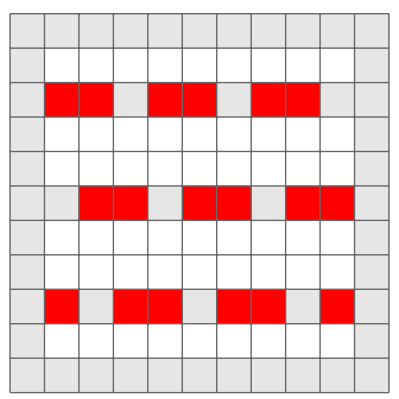

Figure 2: $P_{11} \otimes P_{11}$ where the squares are vertices. Example of the non-occupied attackable vertices in red when only horizontal attacks are considered. The guards occupy a configuration $C_{H}(X)$ where $X=\left(b=2, a_{1}=2, a_{2}=1, a_{3}=a_{\frac{n-2}{3}}=3\right)$, there is one guard at each square in gray, and the white squares contain no guards.

Lemma 4. For any $X \in \mathcal{X}$ and any $v \in A_{H}(X)$, there exists $X^{\prime} \in \mathcal{X}$ such that $v \in C_{H}\left(X^{\prime}\right)$ and the configurations $C_{H}(X)$ and $C_{H}\left(X^{\prime}\right)$ are compatible. That is, in one turn, the guards may move from $C_{H}(X)$ to $C_{H}\left(X^{\prime}\right)$ and defend against an attack at $v$.

Proof. Initially, $\kappa_{H}$ guards are in a configuration $C_{H}(X)$ (see Figure 1). Consider an attack at some vertex $v \in A_{H}(X)$. If $v \in C_{H}(X)$, all guards may remain idle. Hence, let us assume that $v \in A_{H}(X) \backslash C_{H}(X)$.

Let us assume that $v=\left(x_{\ell}(X), y_{w, \ell}(X)-1\right)=\left(x_{\ell}, y_{w, \ell}-1\right)$ for some integers $1 \leq \ell \leq \frac{n-2}{3}$ and $1 \leq w \leq \frac{k-2}{3}$ (note that if $a_{\ell}=1$ then $w>1$ since $v$ is not a border vertex), that is $v$ is to the left of the vertex $\left(x_{\ell}, y_{w, \ell}\right)$ that is occupied by a guard. The cases of attacks at $\left(x_{\ell}(X), y_{w, \ell}(X)+1\right)$ ( $v$ is to the right of an occupied vertex) or $\left(x_{\ell}(X), 2\right)$, or $\left(x_{\ell}(X), k-1\right)$ (the attacked vertex $v$ is adjacent to a border vertex), are similar, by symmetry, to at least one of the two cases below.

The guards will move from the configuration $C_{H}(X)$ to a configuration $C_{H}\left(X^{\prime}\right)$ that defends against the attack at $v$, i.e., $v \in C_{H}\left(X^{\prime}\right)$, where $X^{\prime}=\left\{b^{\prime}, a_{1}^{\prime}, \ldots, a_{\frac{n-2}{3}}^{\prime}\right\}$ as defined below.

Intuitively, for the guards to move from the configuration $C_{H}(\stackrel{3}{X})$ to a configuration $C_{H}\left(X^{\prime}\right)$ that defends against this attack at $v$, all the guards in row $x_{\ell}$ will shift left except for perhaps the guards on the border vertices (it depends on the value of $a_{\ell}$ ). Hence, the only difference between $X$ and $X^{\prime}$ will be the value of the horizontal shift related to row $x_{\ell}$.

Precisely, by the definition of $C_{H}(X)$, there is a guard at $\left(x_{\ell}, y_{w, \ell}\right)$. There are two cases of how the guards will move in response to the attack, depending on the three possible values of $a_{\ell} \in\{1,2,3\}$.

Case i) $a_{\ell} \in\{2,3\}$. To defend against the attack, all the guards in row $x_{\ell}$ except those that occupy border vertices, shift one vertex to the left. That is, the guard at $\left(x_{\ell}, y_{j, \ell}\right)$ moves to $\left(x_{\ell}, y_{j, \ell}-1\right)$ for all $j \in \mathbb{N}^{*}$ such that $1 \leq j \leq \frac{k-2}{3}$. Since the positions of the other guards did not change, the guards occupy a configuration $C_{H}\left(X^{\prime}\right)$ where $b^{\prime}=b, a_{i}^{\prime}=a_{i}$ for all $1 \leq i \leq \frac{n-2}{3}$ such that $i \neq \ell$, but $a_{\ell}^{\prime}=a_{\ell}-1$.

Case ii) $a_{\ell}=1$. To defend against the attack, all the guards in row $x_{\ell}$ except the one at $\left(x_{\ell}, 1\right)$, shift one vertex to the left. That is, the guard at $\left(x_{\ell}, y_{j, \ell}\right)$ moves to $\left(x_{\ell}, y_{j, \ell}-1\right)$ for all $j \in \mathbb{N}^{*}$ such that $1<j \leq \frac{k-2}{3}$. Also, the guard at $\left(x_{\ell}, 2\right)$ jumps to $\left(x_{\ell}, k-1\right)$ which is possible by Lemma 2 and since none of the border guards have to move for any other purpose. Since the 
positions of the other guards did not change, the guards occupy a configuration $C_{H}\left(X^{\prime}\right)$ where $b^{\prime}=b, a_{i}^{\prime}=a_{i}$ for all $1 \leq i \leq \frac{n-2}{3}$ such that $i \neq \ell$, but $a_{\ell}^{\prime}=3$. See Figure 3 .

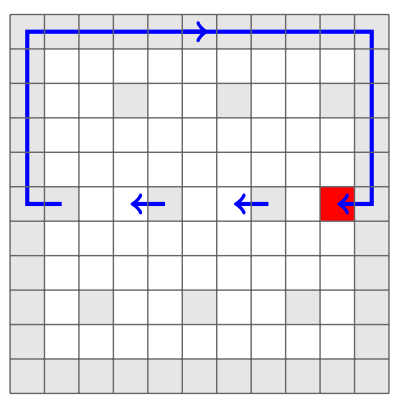

Figure 3: $P_{11} \otimes P_{11}$ where the squares are vertices. Example of an attack in Case ii) at the red square. The guards occupy a configuration $C_{H}(X)$ where $X=\left(b=2, a_{1}=\right.$ $\left.2, a_{2}=1, a_{3}=a_{\frac{n-2}{3}}=3\right)$, there is one guard at each square in gray, and the white squares contain no guards. The arrows (in blue) show the movements of the guards in response to the attack.

\section{$3.2 \quad$ Vertical attacks}

In this section, we consider the entire strong grid $S G_{n \times m}$ partitioned into $\frac{m}{k}$ blocks $S G_{n \times k}$ with block $q$, for $1 \leq q \leq \frac{m}{k}$, being induced by $\{(i, j+(q-1) k) \mid 1 \leq i \leq n, 1 \leq j \leq k\}$. Let us first define a family of parameterised configurations for this graph.

A configuration for the whole grid will be defined as the union of some configurations for each of the $q$ blocks. Formally, for every $1 \leq q \leq \frac{m}{k}$, let us first define:

$$
\mathcal{X}^{q}=\left\{\left(b^{q}, a_{1}^{q}, \ldots, a_{\frac{n-2}{3}}^{q}\right) \mid b^{q} \in\{1,2,3\} \text { and } a_{i}^{q} \in\{1,2,3\} \text { for } i=1, \ldots, \frac{n-2}{3}\right\} .
$$

Given $X^{q}=\left(b^{q}, a_{1}^{q}, \ldots, a_{\frac{n-2}{3}}^{q}\right) \in \mathcal{X}^{q}$, let $x_{i}^{q}\left(X^{q}\right)=3(i-1)+b^{q}+1$, and $y_{j, i}^{q}\left(X^{q}\right)=(q-1) k+$ $3(j-1)+a_{i}^{q}+1$ for every $1 \leq i \leq \frac{n-2}{3}, 1 \leq j \leq \frac{k-2}{3}$, and $1 \leq q \leq \frac{m}{k}$. We set $x_{i}^{q}=x_{i}^{q}\left(X^{q}\right)$ and $y_{j, i}^{q}=y_{j, i}^{q}\left(X^{q}\right)$ when there is no ambiguity.

That is, intuitively, $b^{q}$ will represent the vertical shift of the positions of the guards in configuration $X^{q}$ in the $q^{\text {th }}$ block. Similarly, for every $1 \leq i \leq \frac{n-2}{3}, a_{i}^{q}$ represents the horizontal shift of the positions of the guards in row $x_{i}(X)$ in configuration $X^{q}$ in the $q^{\text {th }}$ block.

Finally, let $\mathcal{Y}=\left\{\left(X^{1}, \ldots, X^{\frac{m}{k}}\right) \mid X^{q} \in \mathcal{X}^{q}\right.$ for $\left.q=1, \ldots, \frac{m}{k}\right\}$.

Vertical Configurations. In order to properly define the following set of configurations, we require the following notation. For a set $S$ of vertices in a configuration $\mathcal{C}$ and an integer $x>0$, let $S^{[x]}$ be the multi-set of vertices that consists of $x$ copies of each vertex in $S$. Intuitively, $S^{[x]}$ will be used to define a configuration where $x$ guards occupy each vertex of $S$. Let us now define the set $\mathcal{C}_{V}$ of configurations as follows.

For every $Y=\left(X^{1}, \ldots, X^{\frac{m}{k}}\right) \in \mathcal{Y}$, let $C_{V}(Y)=B^{\left[\frac{k-2}{3}\right]} \cup \bigcup_{q=1}^{\frac{m}{k}} C_{H}\left(X^{q}\right)$ be the configuration obtained as follows. First, for any $1 \leq q \leq \frac{m}{k}$, guards are placed in configuration $C_{H}\left(X^{q}\right)$ in the 
$q^{\text {th }}$ block. Then, $\frac{k-2}{3}$ guards are added to every border vertex. Note that overall, there are $\frac{k-2}{3}+1$ guards at each vertex of $B$. See an example in Figure 4. Then,

$$
\mathcal{C}_{V}=\left\{C_{V}(Y) \mid Y \in \mathcal{Y}\right\} .
$$

Note that $\left|C_{V}(Y)\right|=\frac{m}{k} \kappa_{H}+2\left(\frac{k-2}{3}\right)(n+m-2)=\kappa_{V}$ for every $Y \in \mathcal{Y}$. That is, any vertical configuration uses $\kappa_{V}$ guards.

Lemma 5. Every configuration $C_{V}(Y) \in \mathcal{C}_{V}$ is a dominating set of $S G_{n \times m}$.

Proof. Since $C_{V}(Y) \in \mathcal{C}_{V}$, by definition, for all $1 \leq q \leq \frac{m}{k}$, there exists $X^{q} \in \mathcal{X}^{q}$ such that the vertices of $C_{H}\left(X^{q}\right)$ are occupied by guards. Therefore, each of the $\frac{m}{k}$ blocks $S G_{n \times k}$ is dominated by the guards within it by Lemma 3 .

In this subsection, we limit the power of the attacker by allowing it to attack only some predefined vertices (this kind of attack will be referred to as a vertical attack). For every configuration $C_{V}(X) \in \mathcal{C}_{V}$ and for any such attack, we show that the guards may be moved (in one turn) in such a way to defend the attacked vertex and reach a new configuration in $\mathcal{C}_{V}$.

Vertical Attacks. Let $Y=\left(X^{1}, \ldots, X^{\frac{m}{k}}\right) \in \mathcal{Y}$ and $C_{V}(Y) \in \mathcal{C}_{V}$. Let

$$
\begin{aligned}
A_{V}(Y)= & \left\{\left(x_{i}^{q}-1, y_{j, i}^{q}\right),\left(x_{i}^{q}+1, y_{j, i}^{q}\right) \mid 1 \leq i \leq \frac{n-2}{3}, 1 \leq j \leq \frac{k-2}{3}, 1 \leq q \leq \frac{m}{k}\right\} \\
& \cup\left\{\left(2, y_{j, n-1}^{q}\right) \mid 1 \leq j \leq \frac{k-2}{3}, 1 \leq q \leq \frac{m}{k}, b^{q}=3\right\} \\
& \cup\left\{\left(n-1, y_{j, 2}^{q}\right) \mid 1 \leq j \leq \frac{k-2}{3}, 1 \leq q \leq \frac{m}{k}, b^{q}=1\right\} .
\end{aligned}
$$

A vertical attack with respect to $Y$ is an attack at any vertex in $A_{V}(Y)$, i.e., an attack at any non-border vertex above or below a guard not on a border vertex. Moreover, if the vertical shift $b^{q}$ of the $q^{\text {th }}$ block equals 3 , then some vertices of the second row of the $q^{\text {th }}$ block may also be attacked (depending on the horizontal shift $a_{n-1}^{q}$ ). Finally, if the vertical shift $b^{q}$ of the $q^{\text {th }}$ block equals 1 , then some vertices of the $(n-1)^{t h}$ row of the $q^{\text {th }}$ block may also be attacked (depending on the horizontal shift $\left.a_{2}^{q}\right)$.

Note that $A_{V}(Y) \cap C_{V}(Y)=\emptyset$, and $A_{V}(Y) \cap A_{H}\left(X^{q}\right)=\emptyset$ for any $X^{q} \in Y$, i.e., any vertical attack with respect to $Y$ is not a horizontal attack with respect to $X^{q} \in Y$ and vice versa. In Figure 5, red squares represent the vertices of $A_{V}(Y)$.

The next lemma proves that, from any vertical configuration and against any vertical attack (with respect to this current configuration), there is a possible strategy for the guards that defends against this attack and leads to a (new) vertical configuration. Therefore, starting from any vertical configuration, there is a strategy of the guards that wins against any sequence of vertical attacks.

Lemma 6. For any $Y \in \mathcal{Y}$ and any $v \in A_{V}(Y)$, there exists $Y^{\prime} \in \mathcal{Y}$ such that $v \in C_{V}\left(Y^{\prime}\right)$ and the configurations $C_{V}(Y)$ and $C_{V}\left(Y^{\prime}\right)$ are compatible. That is, in one turn, the guards may move from $C_{V}(Y)$ to $C_{V}\left(Y^{\prime}\right)$ and defend against an attack at $v$.

Proof. Let $Y=\left(X^{1}, \ldots, X^{\frac{m}{k}}\right)$. Initially, $\kappa_{V}$ guards are in a configuration $C_{V}(Y)$ (see Figure 4 ).

Consider an attack at some vertex $v \in A_{V}(Y)$. Let us assume that $v=\left(x_{\ell}^{z}\left(X^{z}\right)-1, y_{w, \ell}^{z}\left(X^{z}\right)\right)$ for some $1 \leq z \leq \frac{m}{k}, 1 \leq \ell \leq \frac{n-2}{3}$, and $1 \leq w \leq \frac{k-2}{3}$ (note that if $b^{z}=1$, then $\ell>1$ since $v$ is not 


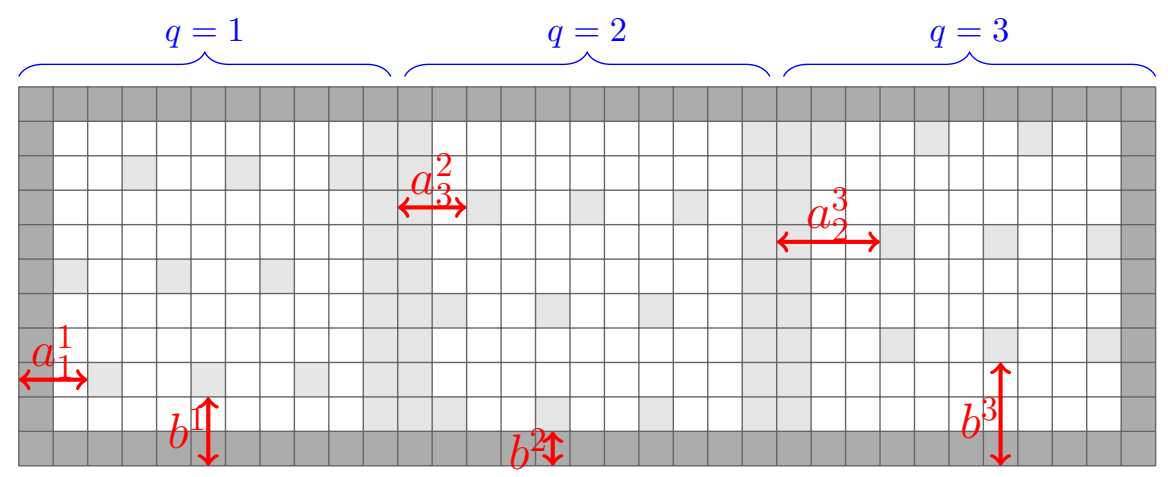

Figure 4: $P_{11} \otimes P_{33}$ where the squares are vertices. Example of a configuration $C_{V}(Y)$ where $k=11, Y=\left(X^{1}, X^{2}, X^{3}\right), X^{1}=(2,2,1,3), X^{2}=(1,1,1,2), X^{3}=(3,3,3,1)$, there are $\frac{k-2}{3}+1=4$ guards at each square in dark gray, 1 guard at each square in light gray, and the white squares contain no guards.

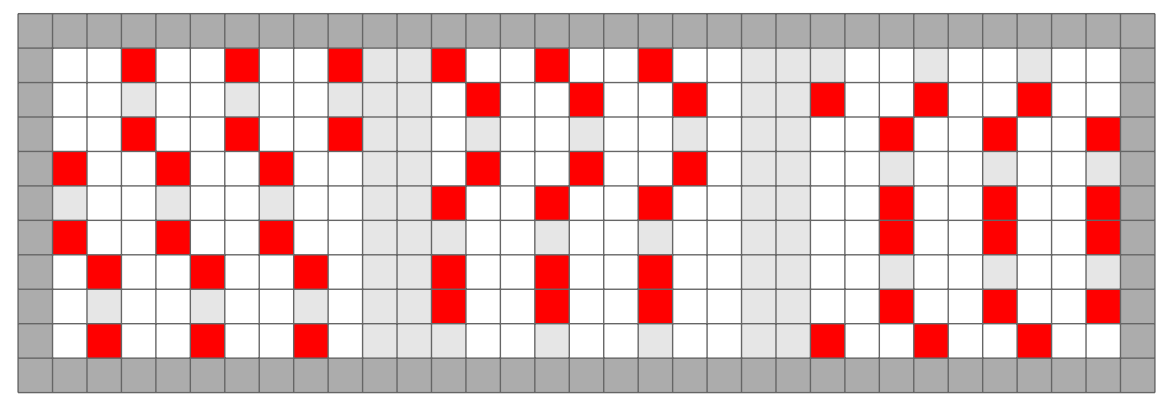

Figure 5: $P_{11} \otimes P_{33}$ where the squares are vertices. Example of the non-occupied attackable vertices in red when only vertical attacks are considered. The guards occupy a configuration $C_{V}(Y)$ where $k=11, Y=\left(X^{1}, X^{2}, X^{3}\right), X^{1}=(2,2,1,3), X^{2}=$ $(1,1,1,2), X^{3}=(3,3,3,1)$, there are $\frac{k-2}{3}+1=4$ guards at each square in dark gray, 1 guard at each square in light gray, and the white squares contain no guards. 
a border vertex). That is, $v$ is a vertex of the $z^{t h}$ block that is below the vertex $\left(x_{\ell}^{z}\left(X^{z}\right), y_{w, \ell}^{z}\left(X^{z}\right)\right)$ which is occupied by a guard.

The cases of attacks at $\left(x_{\ell}^{z}\left(X^{z}\right)+1, y_{w, \ell}^{z}\left(X^{z}\right)\right)\left(v\right.$ is above an occupied vertex), $\left(2, y_{w, n-1}^{z}\left(X^{z}\right)\right)$ ( $v$ is above a border vertex), and $\left(n-1, y_{w, 2}^{z}\left(X^{z}\right)\right)$ ( $v$ is below a border vertex), are similar, by symmetry, to at least one of the two cases below.

The guards will move from the configuration $C_{V}(Y)$ to a configuration $C_{V}\left(Y^{\prime}\right)$ that defends against the attack at $v$, i.e., $v \in C_{V}\left(Y^{\prime}\right)$, where $Y^{\prime}=\left\{X^{\prime 1}, \ldots, X^{\prime \frac{m}{k}}\right\}$ as defined below.

Intuitively, for the guards to move from the configuration $C_{V}(Y)$ to a configuration $C_{V}\left(Y^{\prime}\right)$ that defends against this attack at $v$, all the guards in the block $z$ will shift down except for perhaps the guards on the border vertices (it depends on the value of $b^{z}$ ).

Precisely, by the definition of $C_{V}(Y)$, there is a guard at $\left(x_{\ell}^{z}, y_{w, \ell}^{z}\right)$. There are two cases of how the guards will move in response to the attack, depending on the three possible values of $b^{z} \in\{1,2,3\}$.

Case i) $b^{z} \in\{2,3\}$. To defend against the attack, all the guards in the block $z$ that contains the attacked vertex except those that occupy border vertices of the block $z$, shift one vertex downwards. That is, for all $i, j \in \mathbb{N}$ such that $1 \leq i \leq \frac{n-2}{3}$ and $1 \leq j \leq \frac{k-2}{3}$, the guard at $\left(x_{i}^{z}, y_{j, i}^{z}\right)$ moves to $\left(x_{i}^{z}-1, y_{j, i}^{z}\right)$.

Since the positions of the other guards did not change, the guards occupy a configuration $C_{V}\left(Y^{\prime}\right)$ where $X^{p}=X^{\prime p}$ for all $1 \leq p \leq \frac{m}{k}$ such that $p \neq z$, and $X^{\prime z}=\left(b^{\prime z}, a_{1}^{\prime z}, \ldots, a_{\frac{n-2}{3}}^{\prime z}\right)$ with $a_{i}^{\prime z}=a_{i}^{z}$ for all $1 \leq i \leq \frac{n-2}{3}$, but $b^{\prime z}=b^{z}-1$.

Case ii) $b^{z}=1$. To defend against the attack, all the guards in the block $z$ shift one vertex downwards, except those that occupy the vertices of the border of the block $z$ and the guards just above the bottom border of the block. Using the guards on the border of the (whole) grid, the guards just above the bottom border of the block jump to the row just below the top border of the block $z$.

That is, for all $i, j \in \mathbb{N}$ such that $1<i \leq \frac{n-2}{3}$ and $1 \leq j \leq \frac{k-2}{3}$, the guard at $\left(x_{i}^{z}, y_{j, i}^{z}\right)$ moves to $\left(x_{i}^{z}-1, y_{j, i}^{z}\right)$. Also, the guard at $\left(2, y_{j, i}^{z}\right)$ jumps to $\left(n-1, y_{j, i}^{z}\right)$ which is possible by Lemma 2 since a total of $\frac{k-2}{3}$ guards jump, $\frac{k-2}{3}+1$ guards occupy each vertex of the border of the grid, and since none of the border guards have to move for any other purpose. Since the positions of the other guards did not change, the guards occupy a configuration $C_{V}\left(Y^{\prime}\right)$ where $X^{p}=X^{\prime p}$ for all $1 \leq p \leq \frac{m}{k}$ such that $p \neq z$, and $X^{\prime z}=\left(b^{\prime z}, a_{1}^{\prime z}, \ldots, a_{\frac{n-2}{3}}^{\prime z}\right)$ with $a_{i}^{\prime z}=a_{i}^{z}$ for all $1 \leq i \leq \frac{n-2}{3}$, but $b^{\prime z}=3$. See Figure 6 .

\subsection{Diagonal attacks}

The same $n \times m$ strong grid $S G_{n \times m}$, notations, and configurations for the guards used in subsection 3.2 will be used here.

In this subsection, we limit the power of the attacker by allowing it to attack only some diagonal vertices. For every configuration $C_{V}(X) \in \mathcal{C}_{V}$ and for any such attack, we show that the guards may be moved (in one turn) in such a way to defend the attacked vertex and reach a new configuration in $\mathcal{C}_{V}$. 


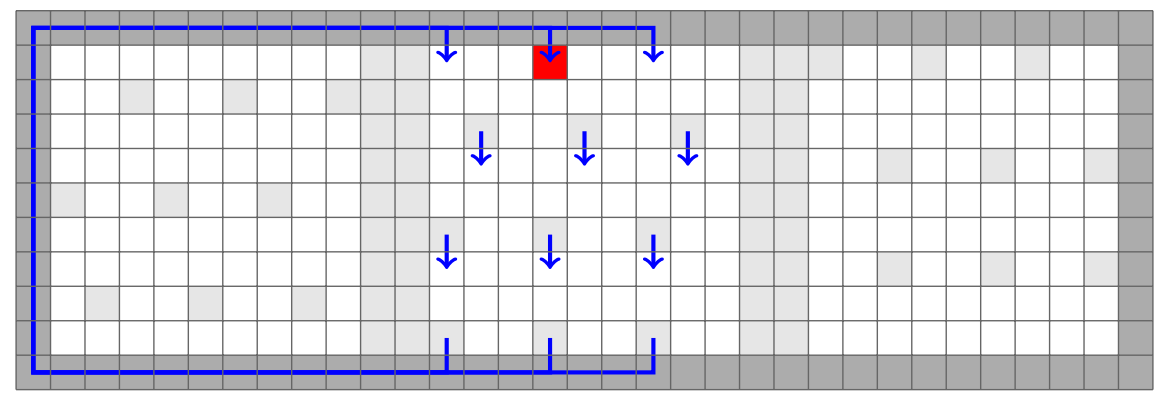

Figure 6: $P_{11} \otimes P_{33}$ where the squares are vertices. Example of an attack in Case iii) at the red square. The guards occupy a configuration $C_{V}(Y)$ where $k=11$, $Y=\left(X^{1}, X^{2}, X^{3}\right), X^{1}=(2,2,1,3), X^{2}=(1,1,1,2), X^{3}=(3,3,3,1)$, there are $\frac{k-2}{3}+1=4$ guards at each square in dark gray, 1 guard at each square in light gray, and the white squares contain no guards. The arrows (in blue) show the movements of the guards in response to the attack.

Diagonal Attacks. Let $Y=\left(X^{1}, \ldots, X^{\frac{m}{k}}\right) \in \mathcal{Y}$ and $C_{V}(Y) \in \mathcal{C}_{V}$. Let $A_{D}(Y)=V\left(S G_{n \times m}\right) \backslash$ $\left(B \cup A_{H}(Y) \cup A_{V}(Y)\right)$. That is, $A_{D}(Y)$ covers all possible attacks that are neither horizontal nor vertical.

A diagonal attack with respect to $Y$ is an attack at any vertex in $A_{D}(Y)$. Note that, for every vertex $v \in A_{D}(Y)$, there is a guard on a vertex adjacent to $v$ and neither in the same column nor in the same row as $v$. In Figure 7 , red squares represent the vertices of $A_{D}(Y)$.

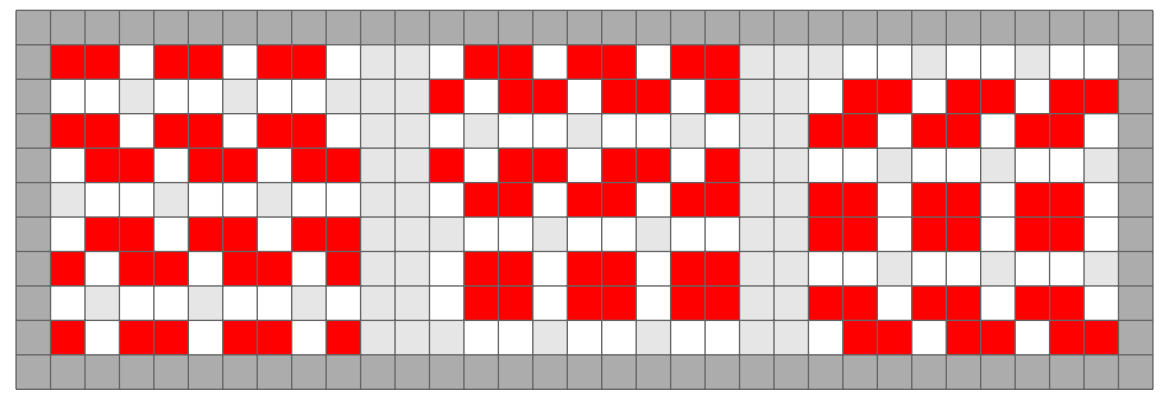

Figure 7: $P_{11} \otimes P_{33}$ where the squares are vertices. Example of the non-occupied attackable vertices in red when only diagonal attacks are considered. The guards occupy a configuration $C_{V}(Y)$ where $k=11, Y=\left(X^{1}, X^{2}, X^{3}\right), X^{1}=(2,2,1,3)$, $X^{2}=(1,1,1,2), X^{3}=(3,3,3,1)$, there are $\frac{k-2}{3}+1=4$ guards at each square in dark gray, 1 guard at each square in light gray, and the white squares contain no guards.

The next lemma proves that, from any vertical configuration and against any diagonal attack (with respect to this current configuration), there is a possible strategy for the guards that defends against this attack and leads to a (new) vertical configuration. Therefore, starting from any vertical configuration, there is a strategy of the guards that wins against any sequence of diagonal attacks.

Lemma 7. For any $Y \in \mathcal{Y}$ and any $v \in A_{D}(Y)$, there exists $Y^{\prime} \in \mathcal{Y}$ such that $v \in C_{V}\left(Y^{\prime}\right)$ and the configurations $C_{V}(Y)$ and $C_{V}\left(Y^{\prime}\right)$ are compatible. That is, in one turn, the guards may move from $C_{V}(Y)$ to $C_{V}\left(Y^{\prime}\right)$ and defend against an attack at $v$. 
Proof. Let $Y=\left(X^{1}, \ldots, X^{\frac{m}{k}}\right)$. Initially, $\kappa_{V}$ guards are in a configuration $C_{V}(Y)$ (see Figure 4).

Consider an attack at some vertex $v \in A_{D}(Y)$. Let us assume that $v=\left(x_{\ell}^{z}\left(X^{z}\right)-1, y_{w, \ell}^{z}\left(X^{z}\right)+1\right)$ for some $1 \leq \ell \leq \frac{n-2}{3}, 1 \leq w \leq \frac{k-2}{3}$, and $1 \leq z \leq \frac{m}{k}$ (note that, if $b^{z}=1$, then $\ell>1$ and if $a_{\ell}^{z}=3$, then $w<\frac{k-2}{3}$ since $v$ is not a border vertex). All other cases are similar by symmetry (see Figures 9 and 10).

The guards will move from a configuration $C_{V}(Y)$ to a configuration $C_{V}\left(Y^{\prime}\right)$ that defends against the attack at $v$, i.e., $v \in C_{V}\left(Y^{\prime}\right)$, where $Y^{\prime}=\left\{X^{\prime 1}, \ldots, X^{\prime \frac{m}{k}}\right\}$ as defined below.

Intuitively, for the guards to move from a configuration $C_{V}(Y)$ to a configuration $C_{V}\left(Y^{\prime}\right)$ that defends against this attack at $v$, in the block $z$ that contains the attacked vertex, the guards in row $x_{\ell}^{z}$ will move as they would in response to a horizontal attack and a vertical attack but simultaneously, so moving diagonally down and to the right, and the remainder of the guards in the block $z$ will move as they would in response to a vertical attack, so moving down.

In particular, if $b^{z}=1$ (there are guards in the row above the bottom border of the block $q$ ), the guards in row 2 in the block $z$ will jump to the row below the top border of the block $z$ using the border of the grid (as specified in Lemma 6). Moreover, if $a_{\ell}^{z}=3$, the guard on vertex $\left(x_{\ell}^{z}, z q-1\right.$ ) jumps to vertex $\left(x_{\ell}^{z}-1, z(q-1)+2\right)$ using the border of the block $z$. So, a total of at most $\frac{k-2}{3}+1$ guards jump which is possible (by Lemma 2) since enough guards are present on each vertex of the border of the grid.

Precisely, after their moves, the guards occupy a configuration $C_{V}\left(Y^{\prime}\right)$ where $X^{p}=X^{\prime p}$ for all $1 \leq p \leq \frac{m}{k}$ such that $p \neq z$, and $X^{\prime z}=\left(b^{\prime z}, a_{1}^{\prime z}, \ldots, a_{\frac{n-2}{3}}^{\prime z}\right)$ with $a_{i}^{\prime z}=a_{i}^{z}$ for all $1 \leq i \leq \frac{n-2}{3}$ such that $i \neq \ell$, but

Case $b^{z} \in\{2,3\}$ and $a_{\ell}^{z} \in\{1,2\}: a_{\ell}^{\prime z}=a_{\ell}^{z}+1$ and $b^{\prime z}=b^{z}-1$.

Case $b^{z} \in\{2,3\}$ and $a_{\ell}^{z}=3: a_{\ell}^{\prime z}=1$ and $b^{\prime z}=b^{z}-1$.

Case $b^{z}=1$ and $a_{\ell}^{z} \in\{1,2\}: a_{\ell}^{\prime z}=a_{\ell}^{z}+1$ and $b^{\prime z}=3$. See Figure 8 .

Case $b^{z}=1$ and $a_{\ell}^{z}=3: a_{\ell}^{\prime z}=1$ and $b^{\prime z}=3$.

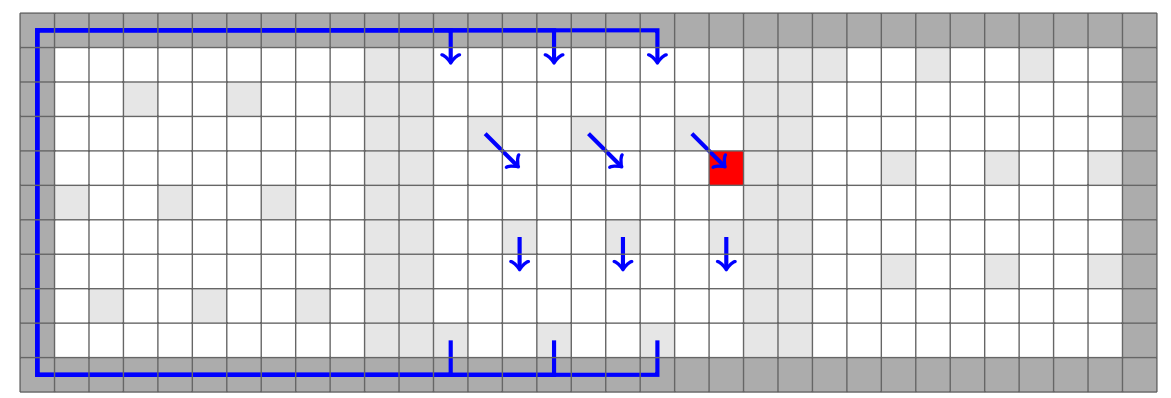

Figure 8: $P_{11} \otimes P_{33}$ where the squares are vertices. Example of a diagonal attack at the red square. The guards occupy a configuration $C_{V}(Y)$ where $k=11, Y=$ $\left(X^{1}, X^{2}, X^{3}\right), X^{1}=(2,2,1,3), X^{2}=(1,1,3,2), X^{3}=(3,3,3,1)$, there are $\frac{k-2}{3}+1=4$ guards at each square in dark gray, 1 guard at each square in light gray, and the white squares contain no guards. The arrows (in blue) show the movements of the guards in response to the attack. 


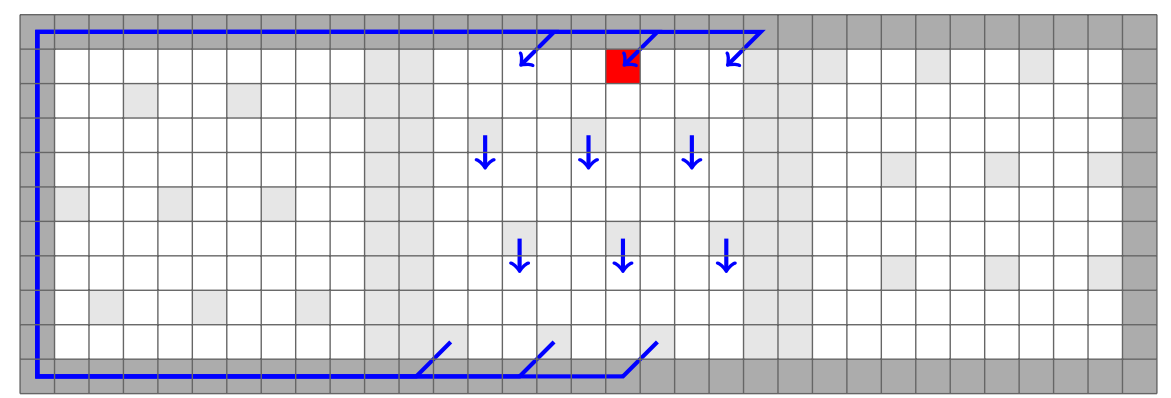

Figure 9: $P_{11} \otimes P_{33}$ where the squares are vertices. Example of a diagonal attack at the red square. The guards occupy a configuration $C_{V}(Y)$ where $k=11, Y=$ $\left(X^{1}, X^{2}, X^{3}\right), X^{1}=(2,2,1,3), X^{2}=(1,1,3,2), X^{3}=(3,3,3,1)$, there are $\frac{k-2}{3}+1=4$ guards at each square in dark gray, 1 guard at each square in light gray, and the white squares contain no guards. The arrows (in blue) show the movements of the guards in response to the attack. To be consistent with the strategy described, the guards in the second row of the middle block move diagonally down and to the left when entering and leaving the border but they may clearly just move vertically down in both instances if they move far enough along the border.

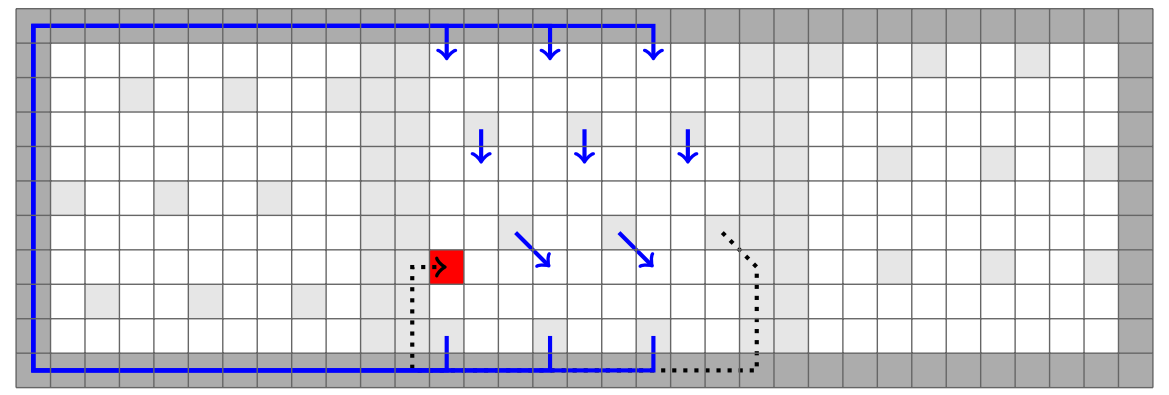

Figure 10: $P_{11} \otimes P_{33}$ where the squares are vertices. Example of a diagonal attack at the red square. The guards occupy a configuration $C_{V}(Y)$ where $k=11, Y=$ $\left(X^{1}, X^{2}, X^{3}\right), X^{1}=(2,2,1,3), X^{2}=(1,1,3,2), X^{3}=(3,3,3,1)$, there are $\frac{k-2}{3}+1=4$ guards at each square in dark gray, 1 guard at each square in light gray, and the white squares contain no guards. The arrows (in blue) show the movements of the guards in response to the attack. The arrow in black is to differentiate between the different guards jumping. 


\subsection{Upper Bound in Strong Grids}

Note that, for any $Y=\left(X^{1}, \ldots, X^{\frac{m}{k}}\right) \in \mathcal{Y}, A_{D}(Y) \cup A_{V}(Y) \cup \bigcup_{q=1}^{\frac{m}{k}} A_{H}\left(X^{q}\right) \cup B=V\left(S G_{n \times m}\right)$. That is, any attack by the attacker in $S G_{n \times m}$ is either an attack at an occupied vertex or a horizontal, vertical or diagonal attack.

Hence, lemmas 4,6, and 7 hold for any possible attack, which leads to our main theorem.

Theorem 8. For all $n, m \in \mathbb{N}^{*}$ such that $m \geq n$,

$$
\gamma_{\text {all }}^{\infty}\left(S G_{n \times m}\right)=\left\lceil\frac{n}{3}\right\rceil\left\lceil\frac{m}{3}\right\rceil+O(m \sqrt{n})=(1+o(1)) \gamma\left(S G_{n \times m}\right) .
$$

Proof. Let $k$ be the integer closest to $\sqrt{n}$ such that $k-2 \equiv 0(\bmod 3)$.

First, we prove that we can restrict our study to the case when $n, m$, and $k$ satisfy the hypothesis of the previous lemmas, i.e., $n-2 \equiv 0(\bmod 3)$ and $m \equiv 0(\bmod k)$. For this purpose, some guards are placed at each of the vertices of a few columns and rows (and these guards will never move) such that what remains to be protected is an $a \times b$ subgrid $H$ such that $a-2 \equiv 0(\bmod 3)$ and $b \equiv 0(\bmod k)$.

If $n-2 \equiv 0(\bmod 3)$, then $a=n$. Otherwise, if $n-2 \equiv 1(\bmod 3)($ resp., 2$)$ then, place one guard at every vertex of the first (resp., the first two) row(s) of $S G_{n \times m}$ and $a=n-1$ (resp., $a=n-2)$. Then, place one guard at every vertex of the $x<k$ first columns of $S G_{n \times m}$, such that $b=m-x$ and $b \equiv 0(\bmod k)$. Overall, $O(m+k n)=O(m+n \sqrt{n})$ guards have been placed, so proving that $\gamma_{\text {all }}^{\infty}(H)=\left\lceil\frac{a}{3}\right\rceil\left\lceil\frac{b}{3}\right\rceil+O(b \sqrt{a})$ will be sufficient to prove the theorem.

Hence, from now on, let us assume that $n$ and $m$ satisfy $n-2 \equiv 0(\bmod 3)$ and $m \equiv 0(\bmod k)$.

Let $Y \in \mathcal{Y}$ be any configuration. The guards initially occupy the configuration $C_{V}(Y)$. By Lemma 5, the guards occupy a dominating set. We show that, for an attack at any vertex $v$, there is $Y^{\prime} \in \mathcal{Y}$ such that $v \in C_{V}\left(Y^{\prime}\right)$ and $C_{V}\left(Y^{\prime}\right)$ is compatible with $C_{V}(Y)$.

Let the attacker attack some unoccupied vertex $v \in V\left(S G_{n \times m}\right)$. As mentioned in Subsection 3.3, the vertex $v$ is in $A_{H}(Y)$ or $A_{V}(Y)$ or $A_{D}(Y)$ (or already contains a guard since every border vertex contains at least one guard). If $v \in C_{V}(Y)$, all guards remain idle. Hence, let us assume that $v \notin C_{V}(Y)$. If $v \in A_{H}\left(X^{q}\right)$ for some $X^{q} \in Y$, then the guards in the block $q$ that contains $v$ will respond as in Lemma 4 (only the guards in the same block and in the same row as $v$ will move, plus some guards on the border of this block if some jump is needed). If $v \in A_{V}(Y)$, then the guards in the block $q$ that contains $v$ will respond as in Lemma 6 . If $v \in A_{D}(Y)$, then the guards in the block $q$ that contains $v$ will respond as in Lemma 7. By Lemma 4, Lemma 6, and Lemma 7, after the attack, the guards occupy a configuration $C_{V}\left(Y^{\prime}\right)$ for some $Y^{\prime} \in \mathcal{Y}$ and thus, can defend against an infinite sequence of attacks.

The above strategy uses $\kappa_{V}=\frac{m}{k}\left(\kappa_{H}\right)+2\left(\frac{k-2}{3}\right)(m+n-2)$ guards (see Subsection 3.2). Since $\kappa_{H}=\frac{(n-2)(k-2)}{9}+2(n+k)-4$ (see Subsection 3.1) and $k=\Theta(\sqrt{n})$, the strategy uses $\kappa_{V}=$ $\left\lceil\frac{n}{3}\right\rceil\left\lceil\frac{m}{3}\right\rceil+O(m \sqrt{n})$ guards, which concludes the proof of the theorem.

\section{Lower Bound in Strong Grids}

So far, the best lower bound for $\gamma_{a l l}^{\infty}\left(S G_{n \times m}\right)$ was the trivial lower bound $\gamma\left(S G_{n \times m}\right)$. In this section, we slightly increase this lower bound, reducing the gap with the new upper bound of the previous section. 
Theorem 9. For all $n, m \in \mathbb{N}^{*}, \gamma_{\text {all }}^{\infty}\left(S G_{n \times m}\right)=\left\lfloor\frac{n}{3}\right\rfloor\left\lfloor\frac{m}{3}\right\rfloor+\Omega(n+m)$.

Proof. $\gamma_{\text {all }}^{\infty}\left(S G_{n \times m}\right)$ is clearly increasing with $n$ and $m$, thus, it is sufficient to prove the theorem for $n \equiv 0(\bmod 3)$ and $m \equiv 0(\bmod 3)$. Hence, let us assume that $n \equiv 0(\bmod 3)$ and $m \equiv 0(\bmod 3)$.

Note that, if $n$ and $m$ are divisible by 3 , there is a unique minimum dominating set of $S G_{n \times m}$ and, in this dominating set, each vertex is dominated by exactly one guard. The idea of the proof is that, in any winning configuration in eternal domination, there are some vertices that are dominated by more than one guard, and/or some guards dominate at most 6 vertices. By double counting, this leads to the necessity of having $\Omega(n+m)$ extra guards compared to the classical domination.

The following claim shows that, whatever be the guards' strategy, at every step, every $4 \times 5$ subgrid that includes 5 border vertices must have at least two guards in it or else the attacker wins.

Claim 10. Consider any configuration of the guards in $S G_{n \times m}$. If there is a $4 \times 5$ subgrid that includes 5 border vertices with only one guard in it, the attacker can win in at most two turns.

Proof of the claim. W.l.o.g. let the $4 \times 5$ subgrid include border vertices from the left column of $S G_{n \times m}$. Also, for some integer $1 \leq x \leq n-4$, let $\{(x, 1), \ldots,(x+4,1)\}$ be the 5 border vertices. If there is only one guard in this subgrid, then the guard must be at $(x+2,2)$ in order to prevent the attacker from winning in one turn as, otherwise, it is not possible to dominate all the vertices of the subgrid. Then, the attacker attacks $(x+2,3)$ which forces the guard at $(x+2,2)$ to move to $(x+2,3)$ as he is the only guard adjacent to that vertex since, initially, there was only one guard in the $4 \times 5$ subgrid. Now the attacker attacks $(x+2,1)$ and wins since every guard is at distance at least 2 from this vertex after the previous moves of the guards since, initially, there was only one guard in the $4 \times 5$ subgrid.

In any configuration $C$, let $x=x(C)$ be the number of $4 \times 5$ subgrids with at least one vertex dominated by two guards and $y=y(C)$ be the number of $4 \times 5$ subgrids where one guard dominates exactly 6 vertices.

Using the previous claim, it can be proved that:

Claim 11. There is $\delta>0$ such that, for any configuration $C$ of the guards in $S G_{n \times m}$ in any winning strategy for the guards, $x+y=\delta(n+m)$ where $x=x(C)$ and $y=y(C)$ are defined as above.

Proof of the claim. Consider the subgraph induced by rows 1 through 4 and columns 6 through $m-5$ of $S G_{n \times m}$.

Considering columns 6 through 13, there must exist a $4 \times 5$ subgrid that includes 5 border vertices and has a guard in its center column as, otherwise, there are no guards in the four center columns of the 8 considered (columns 9 through 12 in this case), which means that $S G_{n \times m}$ is not dominated and hence, this configuration is not part of any winning strategy for the guards. Therefore, by considering the columns eight by eight from the first to the last column in rows 1 through 4 of the subgraph described above, there are at least $\left\lfloor\frac{m-10}{8}\right\rfloor 4 \times 5$ subgrids that fit the profile of the subgrid in Claim 10. Hence, there are at least two guards in each of these subgrids as, otherwise, the attacker wins by Claim 10. Moreover, since there is a guard in the center column of each of these subgrids, there is at least one vertex in each of these subgrids that is dominated by two guards, unless there is a guard on the border in the center column and the other guard(s) are in row 4 . However, in the latter case, the guard on the border in the center column only dominates 6 vertices. By symmetry, this is true for the first and last 4 columns and the topmost 4 rows as 
well. Therefore, there are at least $2\left\lfloor\frac{m-10}{8}\right\rfloor+2\left\lfloor\frac{n-10}{8}\right\rfloor$ subgrids in $S G_{n \times m}$ that fit the profile of the subgrid in Claim 10. Then, $2\left\lfloor\frac{m-10}{8}\right\rfloor+2\left\lfloor\frac{n-10}{8}\right\rfloor \leq x+y$.

Let us consider any winning strategy using $k$ guards. Let $x$ and $y$ be the same as in Claim 11 . At every step, these $k$ guards dominate at most $9 k-3 y$ vertices (with multiplicity, i.e., a vertex is counted once for each guard that dominates it). By the definition of $x$, at least $n m+x$ vertices (with multiplicity) must be dominated. Hence, $9 k-3 y \geq n m+x$. It follows that $k \geq \frac{n m}{9}+\frac{x}{9}+\frac{y}{3}$. By Claim 11, $\frac{x}{9}+\frac{y}{3}=\delta^{\prime}(n+m)$ for some $\delta^{\prime}>0$ and so $k=\frac{n m}{9}+\Omega(n+m)$.

\section{At Most One Guard at each Vertex}

This section is devoted to proving that the two main results presented thus far are also true for the variant of the eternal domination game where at most one guard may occupy a vertex. The corresponding eternal domination number for this variant will be denoted by $\gamma_{a l l}^{* \infty}$. This variant is also considered in, e.g., $[5,17,20]$.

A generalisation of Lemma 2 will be the key to generalising Theorem 8 to this variant of the game. The following definitions are required to properly state this new lemma.

For $t \in \mathbb{N}^{*}$, the set of vertices of the $t$-thick border of $S G_{n \times m}$ is the set

$$
T B_{t}=\bigcup_{1 \leq i \leq n, 1 \leq j \leq m, 1 \leq \ell \leq t}\{(\ell, j),(n+1-\ell, j),(i, \ell),(i, m+1-\ell)\}
$$

and $T B_{0}=\emptyset$. In other words, $T B_{1}=B\left(S G_{n \times m}\right)$ is the border of $S G_{n \times m}$, and $T B_{t}=T B_{t-1} \cup$ $B\left(S G_{n \times m} \backslash T B_{t-1}\right)$ for any $t \geq 1$. Essentially, the $t$-thick border vertices are the vertices of the $t$ leftmost and rightmost columns and the $t$ top and bottom rows of $S G_{n \times m}$.

Recall that $P B=T B_{2} \backslash T B_{1}$ is the set of pre-border vertices. Two vertex-disjoint sets $U, W \subseteq$ $P B$ are said to be non-overlapping, if there is a path $Q$ induced only by vertices of $P B$ such that $U \subseteq V(Q)$ and $V(Q) \cap W=\emptyset$.

Let $P B_{\alpha}=T B_{\alpha+1} \backslash T B_{\alpha}$ be the pre-border vertices of $S G_{n \times m} \backslash\left(T B_{\alpha-1}\right)$.

Lemma 12. Let $\alpha, \beta \in \mathbb{N}^{*}$ such that $\beta \leq \alpha$. Let $U, W \subseteq P B_{\alpha}$ be two non-overlapping subsets of pre-border vertices of $S G_{n \times m} \backslash\left(T B_{\alpha-1}\right)$ such that $|U|=|W|=\beta$. In any configuration $C$ such that $U \subseteq C$, if the $\alpha$-thick border of $S G_{n \times m}$ contains one guard at each of its vertices, then $\beta$ guards may "jump" from $U$ to $W$ in one turn.

Proof. Let $U=\left\{u_{1}, \ldots, u_{\beta}\right\}$ and $W=\left\{w_{1}, \ldots, w_{\beta}\right\}$ where the vertices of $U$ and $W$ are ordered according to the order in which they appear when going clockwise along the cycle induced by $P B_{\alpha}$. Because $\alpha \geq \beta$, and $U$ and $W$ are non-overlapping, there exist vertex-disjoint paths $P_{1}, \ldots, P_{\beta}$ such that, for any $1 \leq i \leq \beta, P_{i}$ is a path from $u_{i}$ to $w_{\beta-i+1}$ whose internal vertices are in $T B_{\alpha}$ (see Figure 11 for an example with $\alpha=\beta=4$ ). Since each vertex in $T B_{\alpha}$ contains one guard, there is a guard at each vertex of the paths $P_{1}, \ldots, P_{\beta}$ except for at the end vertices $w_{1}, \ldots, w_{\beta}$. For the guards to jump from $U$ to $W$, in one turn, for all $1 \leq i \leq \beta$, each guard on each of the vertices of the path $P_{i}$ moves to its neighbour in the direction of $w_{\beta-i+1}$.

Theorem 13. For all $n, m \in \mathbb{N}^{*}$ such that $m \geq n$,

$$
\gamma\left(S G_{n \times m}\right)+\Omega(n+m)=\gamma_{\text {all }}^{* \infty}\left(S G_{n \times m}\right)=\gamma\left(S G_{n \times m}\right)+O(m \sqrt{n}) .
$$




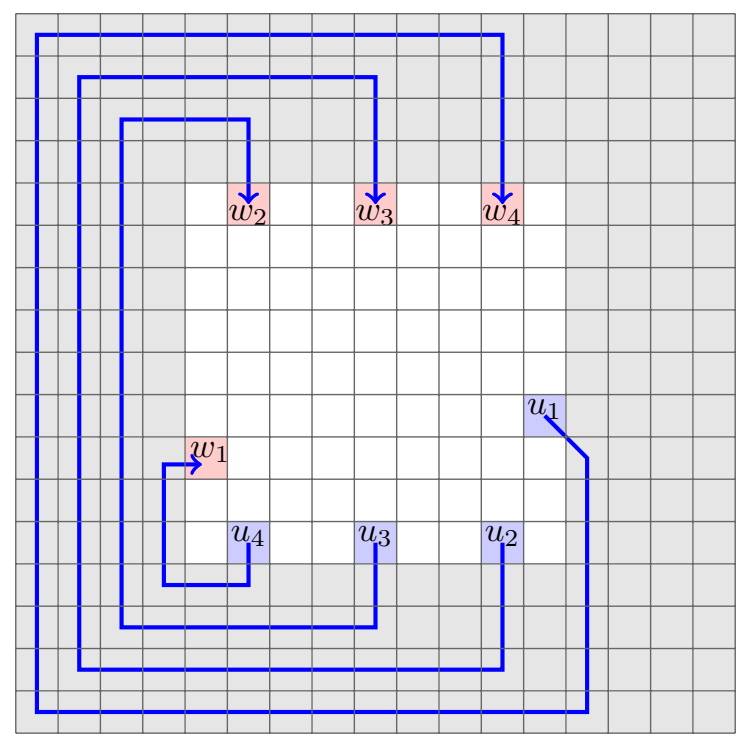

Figure 11: $P_{17} \otimes P_{17}$ where the squares are vertices. Example of how the guards jump in Lemma 12. The vertices of $U=\left\{u_{1}, u_{2}, u_{3}, u_{4}\right\}$ are in blue and the vertices of $W=\left\{w_{1}, w_{2}, w_{3}, w_{4}\right\}$ are in red. Note that $|U|=|W|=\beta=\alpha$. The arrows (in blue) show the vertex-disjoint paths $P_{1}, \ldots, P_{\beta}$ that allow the guards to jump from $U$ to $W$. There is 1 guard at each square in light gray and each vertex of $U$ (in blue), and the white squares contain no guards.

Proof. The lower bound simply follows from Theorem 9 and the fact that $\gamma_{\text {all }}^{* \infty}(G) \geq \gamma_{\text {all }}^{\infty}(G)$ for any graph $G$.

Let us prove the upper bound. The strategy that we propose follows the same principles as the one of Theorem 8 but the border vertices occupied by several guards are replaced by several layers of vertices, each one occupied by a single guard.

Let $k$ be the integer closest to $\sqrt{n}$ such that $k-2 \equiv 0(\bmod 3)$. Let $S G_{n^{*} \times m^{*}}$ be the remaining subgrid that excludes the first and last (topmost and bottommost resp.) $\frac{k-2}{3}$ columns (rows resp.). As in Theorem 8, we may assume that $n=n^{*}+2\left(\frac{k-2}{3}\right)$ and $m=m^{*}+2\left(\frac{k-2}{3}\right)$ are such that $n^{*}-2 \equiv 0(\bmod 3)$ and $m^{*} \equiv 0(\bmod k)$. Indeed, otherwise, it is sufficient to "fill" (place one guard at every vertex) at most two rows and at most $k=O(\sqrt{n})$ columns with one guard per vertex (see proof of Theorem 8).

Hence, from now on, let us assume that $n$ and $m$ satisfy $n-2\left(\frac{k-2}{3}\right)-2 \equiv 0(\bmod 3)$ and $m-2\left(\frac{k-2}{3}\right) \equiv 0(\bmod k)$.

Instead of there being $\frac{k-2}{3}+1$ guards occupying each border vertex of the grid as in Theorem 8 , there is one guard at each vertex of the first $\frac{k-2}{3}+1$ and last $\frac{k-2}{3}+1$ columns and rows.

The strategy for the guards remains the same as the strategy used in Theorem 8 except for when a guard or guards have to jump from one vertex to another, in which case they move as in Lemma 12 with a small exception. The exception is that one of the paths between a vertex being jumped from and a vertex being jumped to in a block $z$, may consist of vertices in one of the columns that forms a border of block $z$. Figure 12 shows an example of a response to a diagonal attack that forces guards to jump and shows that this exception is trivial to deal with. 


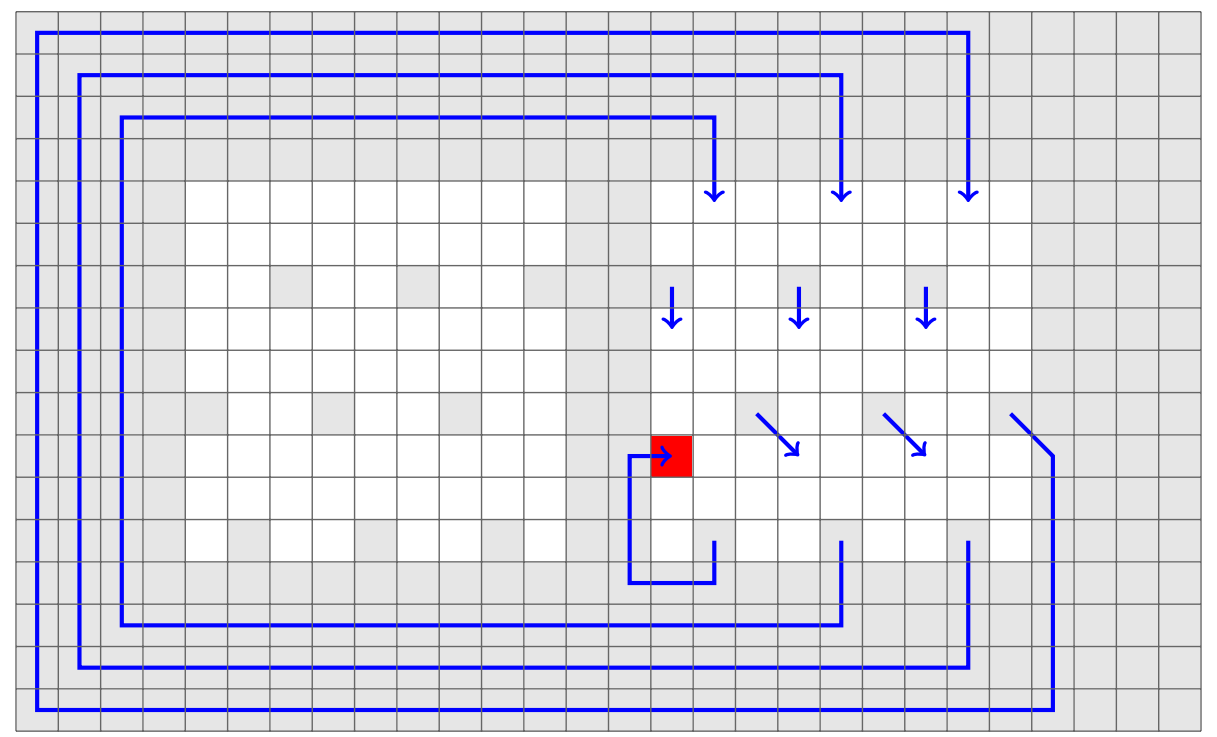

Figure 12: $P_{17} \otimes P_{28}$ where the squares are vertices. Example of a diagonal attack at the red square when at most one guard may occupy a vertex. There is 1 guard at each square in light gray, and the white squares contain no guards. The arrows (in blue) show the movements of the guards in response to the attack.

\section{$6 \quad$ D-Dimensional Grids}

In this section, the eternal domination number of a large family of $D$-dimensional grids is investigated. Intuitively, we consider any graph that is obtained from any vertex-transitive graph that is a supergraph of the $D$-dimensional Cartesian torus and a subgraph of the $D$-dimensional strong torus by "cutting it" (very roughly, just like as a grid can be obtained by "cutting" a torus).

First, we focus on $D$-dimensional Cartesian grids, and then, our algorithm will be generalised for the announced larger graph class.

\subsection{D-dimensional Cartesian grids}

Recall that the Cartesian product of two graphs $G$ and $H$, denoted by $G^{\prime}=G \square H$, is the graph whose vertex set is the Cartesian product of the vertex sets of $G$ and $H$, and two distinct vertices $\left(u_{1}, u_{2}\right) \in V\left(G^{\prime}\right)$ and $\left(v_{1}, v_{2}\right) \in V\left(G^{\prime}\right)$ are adjacent if and only if

- $u_{1}=v_{1}$ and $u_{2}$ is adjacent to $v_{2}$, or

- $u_{2}=v_{2}$ and $u_{1}$ is adjacent to $v_{1}$.

Recall that $P_{n}$ denotes the path with $n>0$ vertices. Let $D \geq 2$ be a fixed integer and $n_{1} \geq n_{2} \geq \cdots \geq n_{D}$ be $D$ positive integers. In this section, let $G=P_{n_{1}} \square \cdots \square P_{n_{D}}$ be the $D$ dimensional grid with corresponding dimensions (note that it is well-defined since the Cartesian product is associative).

This section is devoted to prove the following theorem.

Theorem 14. Let $n_{1} \geq \cdots \geq n_{D}$ be $D=O(1)$ positive integers. For any graph $G=P_{n_{1}} \square \cdots \square P_{n_{D}}$, $\gamma_{\text {all }}^{\infty}(G)=\gamma(G)+o(\gamma(G))$ when $n_{D}=\omega(1)$. 
Notation and informal description of the proof. Every vertex $v$ of $G$ is given with its natural coordinates $\left(v_{1}, \ldots, v_{D}\right)$ where $1 \leq v_{i} \leq n_{i}$ for every $1 \leq i \leq D$ and $u v \in E$ if and only if $\sum_{1 \leq i \leq D}\left|u_{i}-v_{i}\right|=1$. The origin of $G$ is the vertex with coordinates $(1, \ldots, 1)$. For every $1 \leq i \leq D$, the $i$-face (resp., the $-i$-face) of $G$ is the $(D-1)$-dimensional grid induced by the vertices $v$ of $G$ such that $v_{i}=n_{i}$ (resp., $v_{i}=1$ ). The border of $G$ is the set of its vertices with degree $<2 D$ or, equivalently, the set of the vertices belonging to some face of $G$. The $i$-neighbour (resp., $-i$-neighbour) of $v$ is the vertex $u \in N(v)$ such that $u_{i}-v_{i}=1$ (resp., $v_{i}-u_{i}=1$ ), if it exists.

Let $C_{n}$ denote the cycle with $n>0$ vertices and let $G_{T}=C_{n_{1}} \square \cdots \square C_{n_{D}}$ be the $D$-dimensional torus. Note that $\gamma(G)=\Theta\left(\gamma\left(G_{T}\right)\right)$ and $\gamma_{\text {all }}^{\infty}\left(G_{T}\right)=\gamma\left(G_{T}\right)=\Theta\left(\prod_{1 \leq i \leq D} n_{i}\right)$ (the last equality holds since $D$ is a fixed constant). The latter is easy to see since $G_{T}$ is vertex-transitive, and so, if all the guards move in the exact same direction at each turn, then they maintain that they occupy a dominating set, and, one of these directions corresponds to defending against the attack at that turn since the guards occupy a dominating set. In particular, an eternal dominating strategy for $G_{T}$ consists of the following. First, one guard is placed at every vertex of a minimum dominating set of $G_{T}$. Now, when a non-occupied vertex $u$ is attacked, let $v \in N(u)$ be any vertex occupied by a guard and, w.l.o.g., say that $u$ is the $i$-neighbour of $v$ for some $1 \leq i \leq D$ (where the notion of $i$-neighbour must be understood "modulo $n_{i}$ "), then all guards move toward their $i$-neighbour (since $G_{T}$ is vertex-transitive, the set of the positions of the guards remains a dominating set). In what follows, such a strategy will be "simulated" in the Cartesian grid.

The proof of the above theorem is the description of a strategy that is given throughout this section. The key idea is to recursively cover $G$ with smaller $D$-dimensional grids (called blocks) as we did for the 2-dimensional grid in Section 3. Intuitively, for any attack at some vertex $v \in V(G)$, an adequate block will be considered as a torus (by using an adaptation of Lemma 2) and the guards in it will move in order to protect the attacked vertex and maintain a dominating set in $G$.

Recursive cover into (moving) blocks. For all $1 \leq i \leq D$, let $m_{i}=2^{i-1}(D-1)+1$, and let $n_{D}^{\frac{1}{m_{D}}}$ be an integer such that $n_{i} \equiv 0\left(\bmod n_{D}^{\frac{1}{m_{D}}}\right)$ for all $1 \leq i \leq D$ (here, we assume that such an integer exists and it will be shown in the proof of Theorem 14 how to reach this situation). Let $G$ be the unique $(D+1)$-block (with origin $(1, \cdots, 1)$ ). Intuitively, the $(D+1)$-block $G$ is partitioned into $D$-dimensional grids, called $D$-blocks, of dimension $n_{D}^{\frac{m_{1}}{m_{D}}} \times n_{D}^{\frac{m_{2}}{m_{D}}} \times \cdots \times n_{D}^{\frac{m_{D}}{m_{D}}}$, and then, for every $1 \leq i<D$, any $(i+1)$-block will be cut into "slices" of "width" $n_{D}^{\frac{1}{m_{D}}}$ (the $i$-blocks) along the $(i+1)^{t h}$ dimension. The following property of the $m_{i}$ is important to note. For all $2 \leq j \leq D$,

$$
\begin{aligned}
\sum_{a=1}^{j-1} m_{a} & =j-1+\frac{D-1}{2} \sum_{a=1}^{j-1} 2^{a} \\
& =j-1+\frac{D-1}{2}\left(\frac{1-2^{j}}{1-2}-1\right) \\
& =j-1+2^{j-1}(D-1)-D+1 \\
& =m_{j}+j-D-1 .
\end{aligned}
$$


Precisely, for every $1 \leq i \leq D$, an $i$-block with origin $v$ is the subgraph of $G$ which is a $D$ dimensional grid of dimensions $\left(n_{i, 1}, \ldots, n_{i, D}\right)=\left(n_{D}^{\frac{m_{1}}{m_{D}}} \times \cdots \times n_{D}^{\frac{m_{i}}{m_{D}}} \times n_{D}^{\frac{1}{m_{D}}} \times \cdots \times n_{D}^{\frac{1}{m_{D}}}\right)$ induced by the vertices $u$ such that $v_{j} \leq u_{j} \leq v_{j}+n_{i, j}-1$ for all $1 \leq j \leq D$. Initially, for all $1<i \leq D$, any $i$-block $B$ with origin $\left(v_{1}, \ldots, v_{D}\right)$ is partitioned into $\frac{n_{i, i}}{n_{D}^{\frac{1}{m_{D}}}}=n_{D}^{\frac{m_{i}-1}{m_{D}}}$ blocks that are $(i-1)$-blocks with origins $v^{(j)}$ with coordinates $\left(v_{1}, \ldots, v_{i-1}, v_{i}^{(j)}=v_{i}+j n_{D}^{\frac{1}{m_{D}}}, v_{i+1}, \ldots, v_{D}\right)$ for $0 \leq j<n_{D}^{\frac{m_{i}-1}{m_{D}}}$. That is, $B$ is partitioned into $n_{D}^{\frac{m_{i}-1}{m_{D}}}(i-1)$-blocks such that only vertices of the $i$ or $-i$-faces of two such $(i-1)$-blocks may be adjacent (see Figure 13 (top)). The $(D+1)$-block $G$ is partitioned into $\frac{n_{1} \ldots n_{D}}{\prod_{j=1}^{D} n_{D}^{\frac{m_{j}}{m_{D}}}}=\frac{n_{1} \ldots n_{D-1}}{n_{D}^{\frac{m_{D}-1}{m_{D}}}}$ (by (1)) non-intersecting $D$-blocks.

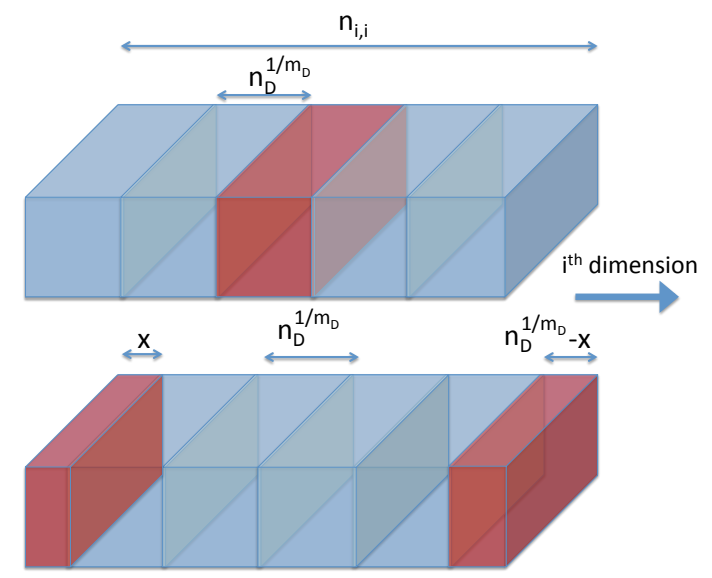

Figure 13: Schematic representation of moving $(i-1)$-blocks inside an $i$-block.

As it will become clearer soon, the blocks will be defined by the positions of the guards. We refer to the guards related to any $i$-block as the $i$-level guards (to be precised below). In particular, an $i$-block $B^{\prime}$ will not have a fixed position (given by its origin) but, when all $j$-level guards inside $B^{\prime}$ will move (for every $j \leq i$ ), the corresponding $i$-block $B^{\prime}$ will move accordingly. Precisely, an $(i-1)$-block $B$ may only move along the $i^{\text {th }}$-dimension inside the $i$-block $B^{\prime}$ containing it, meaning that the origin $v$ of $B$ may move to its $i$-neighbour or to its $-i$-neighbour (i.e., all vertices of $B$ are translated by one unit along their $i^{\text {th }}$ coordinate, either positively or negatively). This leads to a subtlety when an $(i-1)$-block reaches the $i$-face (resp., the $-i$-face) of the $i$-block containing it. To deal with this difficulty, let us extend the notion of blocks. To simplify things, assume that the origin $v$ of an $i$-block $B^{\prime}$ is such that $v_{j}+n_{i, j}-1 \leq n_{j}$ for all $1 \leq j \leq D$, i.e., the subgraph induced by the vertices of $B^{\prime}$ is connected. If the origin $u$ of an $(i-1)$-block $B$ inside such an $i$-block $B^{\prime}$ with origin $v$ is such that $v_{i}+n_{D}^{\frac{m_{i}}{m_{D}}}<u_{i}+n_{D}^{\frac{1}{m_{D}}}$, then the block $B$ is the subgraph induced by the union of the two $D$-dimensional grids with vertices $w \in B^{\prime}$ with coordinates that satisfy $u_{j} \leq w_{j} \leq u_{j}+n_{i, j}-1$ for all $1 \leq j \leq D$ such that $j \neq i$, and either $u_{i} \leq w_{i} \leq v_{i}+n_{D}^{\frac{m_{i}}{m_{D}}}$ or $v_{i} \leq w_{i} \leq u_{i}-n_{D}^{\frac{m_{i}}{m_{D}}}+n_{D}^{\frac{1}{m_{D}}}$ (see Figure 13 (bottom)). The $+i$-face of $B$ then consists of the vertices $w \in B^{\prime}$ such that $w_{i}=u_{i}-n_{D}^{\frac{m_{i}}{m_{D}}}+n_{D}^{\frac{1}{m_{D}}}$, and the $-i$-face of $B$ then consists of the vertices 
$w \in B^{\prime}$ such that $w_{i}=u_{i}$. Note that, since the "division" of blocks is recursive, a block may actually consist of several connected components but, intuitively, it remains a $D$-dimensional grid cut according to several hyperplanes (see Figure 14).

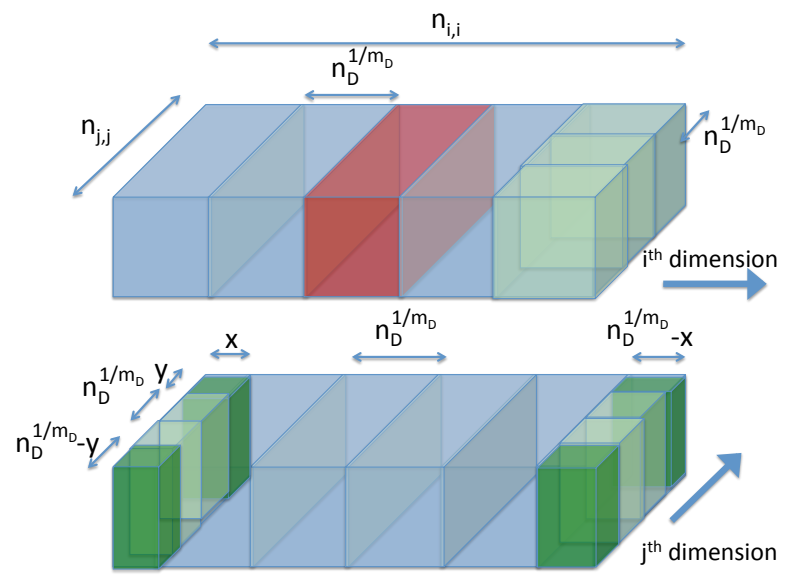

Figure 14: Schematic representation of recursive division of blocks.

The following result is a direct adaptation of Lemma 2 to this new context. Intuitively, it says that the guards in an $(i-1)$-block which is part of a larger $i$-block $B$ move in $B$ as if $B$ was a $D$-dimensional torus. As before, this will be the key to the correctness of the forthcoming strategy. Let $S_{i}$ be the number of vertices of an $i$-face of an $i$-block.

Lemma 15. For any $1 \leq i \leq D$, let $B^{\prime}$ be any $(i+1)$-block, $B$ be any $i$-block inside $B^{\prime}$, and assume that every non-border vertex of $B$ (any vertex in $B$ that is not a vertex of a face of $B$ ) is occupied by at most $x$ guards. Moreover, assume that every vertex of the $\pm(i+1)$-faces and of the $\pm i$-faces of $B^{\prime}$ is occupied by at least $x S_{i}$ guards. Then, for any number of vertices of $B$ adjacent to the $i$-face of $B$, all the guards occupying each of those vertices may "jump" to any vertex of the - $i$-face of $B$ in one step. Moreover, only the guards "jumping" and those on the border of $B^{\prime}$ move.

The strategy. We are now ready to formally define the strategy for eternally dominating the $D$ dimensional Cartesian grid $G$. Let us partition $G$ into $\frac{n_{1} \ldots n_{D-1}}{n_{D}^{\frac{m_{D}-1}{m_{D}}}} D$-blocks and, for every $1 \leq i \leq D$, let us recursively partition each $i$-block into $n_{D}^{\frac{m_{i}-1}{m_{D}}}(i-1)$-blocks (initially, there are no "cut" blocks, i.e., every block is a connected $D$-dimensional subgraph of $G$ ).

Recall that $S_{i}$ denotes the number of vertices of an $i$-face of an $i$-block.

Claim 16. For all $2 \leq i \leq D, S_{i}=n_{i, 1} \ldots n_{i, i-1} * 1 * n_{i, i+1} \ldots n_{i, D}=n_{i, 1} \ldots n_{i, i-1} n_{D}^{\frac{D-i}{m}}$, i.e.,

$$
S_{i}=n_{D}^{\frac{D-i}{m}} \prod_{j=1}^{i-1} n_{D}^{\frac{m_{j}}{m_{D}}}
$$

and $S_{1}=1 * n_{1,2} \ldots n_{1, D}=n_{D}^{\frac{D-1}{m_{D}}}$. Moreover, for $1 \leq j<D$, a largest face of a $j$-block has $n_{j, 1} \ldots n_{j, j} n_{D}^{\frac{D-j-1}{m_{D}}}$ vertices, and a largest face of a $D$-block has $n_{D, 2} \ldots n_{D, D}$ vertices. 
The initial configuration of the guards can be broken down into three parts as follows. For the first part, for each 1-block in $G$, we place guards of level 1 at the vertices of a minimum dominating set of that 1-block, where all the vertices of each 1-block must be dominated by guards at vertices in the same 1-block. Let $S_{0}=g_{0}=0$ and, for all $1 \leq i \leq D$, let $g_{i}=S_{i}+g_{i-1} S_{i-1}$. Then, for the second part, for $i=1$ to $D$, place $g_{i}$ guards of level $i$ at each of the vertices of the $\pm i$-faces of the $i$-blocks. Intuitively, $g_{i}$ represents the maximum number of guards of level $\leq i$ occupying a vertex.

Lastly, for the third part, place guards on each of the border vertices of $G$, so that each of these border vertices is occupied by $g_{D}$ guards (note that some of these border vertices already had guards occupying them from the second part).

Lemma 17. The strategy uses a total number of $\gamma(G)+o(\gamma(G))$ guards.

Proof. First, recall that $\gamma(G)=\Theta\left(n_{1} \ldots n_{D}\right)$.

For the first part: there are $\gamma(G)+o(\gamma(G))$ guards. We will show this by proving that a guard at each vertex of a minimum dominating set of $G$ plus one guard at each border vertex of each 1-block in $G$ uses $\gamma(G)+o(\gamma(G))$ guards and that is more guards than one guard at each vertex of a minimum dominating set of each 1-block in $G$. Indeed, to see that it uses more guards, simply observe that any non-border vertex of a 1-block cannot dominate any vertex in another 1-block.

We now prove that placing a guard at each vertex of a minimum dominating set of $G$ plus one guard at each border vertex of each 1-block in $G$ uses $\gamma(G)+o(\gamma(G))$ guards. Hence, for this part, all that remains to prove is that the total number of vertices of the borders of the 1-blocks is $o(\gamma(G))$. First, note that each block has $2 D(D-1)$-dimensional faces which constitute its border vertices, and, for each 1-block, each of these faces has at most $n_{D}^{\frac{m_{1}}{m_{D}}} n_{D}^{\frac{D-2}{m_{D}}}=n_{D}^{\frac{2 D-2}{m_{D}}}$ vertices (by Claim 16). Now, recall that, for all $1<i \leq D$, each $i$-block contains $n_{D}^{\frac{m_{i}-1}{m_{D}}}(i-1)$-blocks. Therefore, since $\frac{n_{1} \ldots n_{D-1}}{\frac{m_{D}-1}{n_{D}}}$ is the number of $D$-blocks in $G$, then $\left(\frac{n_{1} \ldots n_{D-1}}{n_{D}^{\frac{m_{D}-1}{m_{D}}}}\right) \prod_{\ell=1}^{D-1} n_{D}^{\frac{m_{\ell+1}-1}{m_{D}}}$ is the number of 1-blocks in $G$. Thus, the total number of vertices of the borders of the 1-blocks is at most

$$
\begin{aligned}
2 D * n_{D}^{\frac{2 D-2}{m_{D}}}\left(\frac{n_{1} \ldots n_{D-1}}{n_{D}^{\frac{m_{D}-1}{m_{D}}}}\right) \prod_{\ell=1}^{D-1} n_{D}^{\frac{m_{\ell+1}-1}{m_{D}}} & =2 D * n_{D}^{\frac{2 D-2}{m_{D}}}\left(\frac{n_{1} \ldots n_{D-1}}{n_{D}^{\frac{m_{D}-1}{m_{D}}}}\right)\left(n_{D}^{\frac{-(D-1)}{m_{D}}}\right) \prod_{\ell=2}^{D} n_{D}^{\frac{m_{\ell}}{m_{D}}} \\
& =2 D * n_{D}^{\frac{D-1}{m_{D}}}\left(\frac{n_{1} \ldots n_{D-1}}{n_{D}^{\frac{m_{D}-1}{m_{D}}}}\right)\left(\frac{n_{D} n_{D}^{\frac{m_{D}-1}{m_{D}}}}{n_{D}^{\frac{m_{1}}{m_{D}}}}\right) \\
& =2 D n_{1} \ldots n_{D-1} n_{D}^{\frac{m_{D}-1}{m_{D}}} \\
& =o(\gamma(G)) \quad(\text { since } D=O(1)) .
\end{aligned}
$$

Hence, for the first part, there are at most $\gamma(G)+o(\gamma(G))$ guards.

For the second part: first, we prove by induction on $i$ that, for all $0 \leq i \leq D, g_{i} \leq i S_{i}$. It is trivially true for $i=0$ and $i=1$ (since $g_{1}=S_{1}$ ). Then, for the inductive hypothesis, for some $1<k<D$, suppose that $g_{k} \leq k S_{k}$. Then, 


$$
\begin{aligned}
g_{k+1} & =S_{k+1}+g_{k} S_{k} \\
& \leq S_{k+1}+k S_{k}^{2} \quad(\text { by the inductive hypothesis) } \\
& =S_{k+1}+k\left(n_{D}^{\frac{D-k}{m}} \prod_{j=1}^{k-1} n_{D}^{\frac{m_{j}}{m_{D}}}\right)^{2} \quad \text { (by Claim 16) } \\
& =S_{k+1}+k\left(n_{D}^{\frac{D-k}{m_{D}}} n_{D}^{\frac{m_{k}+k-D-1}{m_{D}}}\right)^{2} \quad \text { (by (1)) } \\
& =S_{k+1}+k n_{D}^{\frac{2 m_{k}-2}{m_{D}}} \\
& =S_{k+1}+k n_{D}^{\frac{D-k-1}{m_{D}}} n_{D}^{\frac{m_{k}+m_{k}+k-D-1}{m_{D}}} \\
& =S_{k+1}+k n_{D}^{\frac{D-k-1}{m_{D}}} \prod_{j=1}^{k} n_{D}^{\frac{m_{j}}{m_{D}}} \quad \text { (by (1)) } \\
& =S_{k+1}+k S_{k+1} \quad(\text { by Claim 16) } \\
& =(k+1) S_{k+1} . \quad
\end{aligned}
$$

Thus, for all $0 \leq i \leq D$,

$$
g_{i} \leq i S_{i}
$$

Then, for the second part, there are

$$
\sum_{i=1}^{D-1}\left(2 g_{i} S_{i}\left(\frac{n_{1} \ldots n_{D-1}}{n_{D}^{\frac{m_{D}-1}{m_{D}}}}\right) \prod_{\ell=i}^{D-1} n_{D}^{\frac{m_{\ell+1}-1}{m_{D}}}\right)+2 g_{D} S_{D}\left(\frac{n_{1} \ldots n_{D-1}}{n_{D}^{\frac{m_{D}-1}{m_{D}}}}\right)
$$

guards. Note that: 1) $2 g_{i} S_{i}$ corresponds to the number of guards on the $\pm i$-faces of the $i$-block, 2) $\frac{n_{1} \ldots n_{D-1}}{\frac{m_{D}-1}{n_{D}}}$ is the number of $D$-blocks in $G$, and 3) $\left(\frac{n_{1} \ldots n_{D-1}}{\frac{m_{D}-1}{m_{D}}}\right) \prod_{\ell=i}^{D-1} n_{D}^{\frac{m_{\ell+1}-1}{m_{D}}}$ is the number of $i$-blocks in $G$ for all $1 \leq i \leq D-1$. Since $D$ is a fixed constant, to show that the sum above is $o(\gamma(G))$, it suffices to show that, for all $1 \leq i \leq D-1$, the summand is $o(\gamma(G))$, and that $2 g_{D} S_{D}\left(\frac{n_{1} \ldots n_{D-1}}{\frac{m_{D}-1}{n_{D}}}\right)=o(\gamma(G))$. We begin by showing the latter is true: 


$$
\begin{aligned}
2 g_{D} S_{D}\left(\frac{n_{1} \ldots n_{D-1}}{n_{D}^{\frac{m_{D}-1}{m_{D}}}}\right) & \leq 2 D S_{D}^{2}\left(\frac{n_{1} \ldots n_{D-1}}{n_{D}^{\frac{m_{D}-1}{m_{D}}}}\right) \quad(\text { by }(2)) \\
& =2 D\left(n_{D}^{\frac{m_{D}-1}{m_{D}}}\right)^{2}\left(\frac{n_{1} \ldots n_{D-1}}{n_{D}^{\frac{m_{D}-1}{m_{D}}}}\right) \quad(\text { by Claim } 16 \text { and }(1)) \\
& =2 D n_{1} \ldots n_{D-1} n_{D}^{\frac{m_{D}-1}{m_{D}}} \\
& =o(\gamma(G)) \quad(\text { since } D=O(1)) .
\end{aligned}
$$

Now, we show the former holds for all $2 \leq i \leq D-1$. That is, for all $2 \leq i \leq D-1$,

$$
\begin{aligned}
& 2 g_{i} S_{i}\left(\frac{n_{1} \ldots n_{D-1}}{n_{D}^{\frac{m_{D}-1}{m_{D}}}}\right) \prod_{\ell=i}^{D-1} n_{D}^{\frac{m_{\ell+1}-1}{m_{D}}} \\
& \left.\leq 2 i S_{i}^{2}\left(\frac{n_{1} \ldots n_{D-1}}{n_{D}^{\frac{m_{D}-1}{m_{D}}}}\right) \prod_{\ell=i}^{D-1} n_{D}^{\frac{m_{\ell+1}-1}{m_{D}}} \quad \text { by }(2)\right) \\
& =2 i\left(n_{D}^{\frac{D-i}{m_{D}}} \prod_{j=1}^{i-1} n_{D}^{\frac{m_{j}}{m_{D}}}\right)^{2}\left(\frac{n_{1} \ldots n_{D-1}}{n_{D}^{\frac{m_{D}-1}{m_{D}}}}\right) \prod_{\ell=i}^{D-1} n_{D}^{\frac{m_{\ell+1}-1}{m_{D}}} \quad \text { (by Claim 16) } \\
& =2 i\left(n_{D}^{\frac{D-i}{m_{D}}} \prod_{j=1}^{i-1} n_{D}^{\frac{m_{j}}{m_{D}}}\right)^{2}\left(n_{D}^{\frac{-(D-i)}{m_{D}}} \prod_{\ell=i+1}^{D} n_{D}^{\frac{m_{\ell}}{m_{D}}}\right)\left(\frac{n_{1} \ldots n_{D-1}}{n_{D}^{\frac{m_{D}-1}{m_{D}}}}\right) \\
& =2 i\left(\prod_{j=1}^{D} n_{D}^{\frac{m_{j}}{m_{D}}}\right)\left(\frac{n_{1} \ldots n_{D-1}}{n_{D}^{\frac{m_{D}+m_{i}-1}{m_{D}}}}\right)\left(n_{D}^{\frac{D-i}{m_{D}}} \prod_{j=1}^{i-1} n_{D}^{\frac{m_{j}}{m_{D}}}\right) \\
& =2 i\left(n_{D} n_{D}^{\frac{m_{D}-1}{m_{D}}}\right)\left(\frac{n_{1} \ldots n_{D-1}}{n_{D}^{\frac{m_{D}+m_{i}-1}{m_{D}}}}\right)\left(n_{D}^{\frac{D-i}{m_{D}}} n_{D}^{\frac{m_{i}+i-D-1}{m_{D}}}\right) \\
& =
\end{aligned}
$$

Lastly, we deal with the case of the former for $i=1$ : 


$$
\begin{aligned}
2 g_{1} S_{1}\left(\frac{n_{1} \ldots n_{D-1}}{n_{D}^{\frac{m_{D}-1}{m_{D}}}}\right) \prod_{\ell=1}^{D-1} n_{D}^{\frac{m_{\ell+1}-1}{m_{D}}} & =2\left(n_{D}^{\frac{D-1}{m_{D}}}\right)^{2}\left(\frac{n_{1} \ldots n_{D-1}}{n_{D}^{\frac{m_{D}-1}{m_{D}}}}\right) \prod_{\ell=1}^{D-1} n_{D}^{\frac{m_{\ell+1}-1}{m_{D}}} \quad \text { (by Claim 16) } \\
& =2\left(n_{D}^{\frac{D-1}{m_{D}}}\right)^{2}\left(n_{D}^{\frac{-(D-1)}{m_{D}}} \prod_{\ell=2}^{D} n_{D}^{\frac{m_{\ell}}{m_{D}}}\right)\left(\frac{n_{1} \ldots n_{D-1}}{n_{D}^{\frac{m_{D}-1}{m_{D}}}}\right) \\
& =2\left(\prod_{\ell=2}^{\frac{D}{m_{D}}}\right)\left(\frac{n_{1} \ldots n_{D-1}}{n_{D}^{\frac{m_{D}-1}{m_{D}}}}\right)\left(n_{D}^{\frac{D-1}{m_{D}}}\right) \\
& \left.=2\left(\frac{n_{D} n_{D}^{\frac{m_{D}-1}{m_{D}}}}{n_{D}^{\frac{m_{1}}{m_{D}}}}\right)\left(\frac{n_{1} \ldots n_{D-1}}{n_{D}^{\frac{m_{D}-1}{m_{D}}}}\right)\left(n_{D}^{\frac{D-1}{m_{D}}}\right) \quad \quad \text { (by }(1)\right) \\
& =2 n_{1} \ldots n_{D-1} n_{D}^{\frac{m_{D}-m_{1}+D-1}{m_{D}}} \\
& =2 n_{1} \ldots n_{D-1} n_{D}^{\frac{m_{D}-1}{m_{D}}} \\
& =o(\gamma(G))
\end{aligned}
$$

Therefore, for the second part, there are $o(\gamma(G))$ guards.

For the third part: first, note that, like for the first part, $G$ has $2 D(D-1)$-dimensional faces which constitute its border vertices, and each of these faces has at most $n_{1} \ldots n_{D-1}$ vertices. Then, for the third part, there are at most

$$
\begin{aligned}
2 D g_{D}\left(n_{1} \ldots n_{D-1}\right) & \leq 2 D^{2} S_{D}\left(n_{1} \ldots n_{D-1}\right) \quad(\text { by }(2)) \\
& =2 D^{2} n_{1} \ldots n_{D-1} n_{D}^{\frac{m_{D}-1}{m}} \quad(\text { by Claim } 16 \text { and }(1)) \\
& =o(\gamma(G)) \quad(\text { since } D=O(1))
\end{aligned}
$$

guards. Since the first part adds $\gamma(G)+o(\gamma(G))$ guards, and each of the other two parts adds at most $o(\gamma(G))$ guards, then, the total number of guards is $\gamma(G)+o(\gamma(G))$.

Now, let us assume that an unoccupied vertex $u$ is attacked and let $v \in N(u)$ be its occupied neighbour (exists by induction hypothesis on the number of attacks). W.l.o.g., let us assume that $u$ is the $i$-neighbor of $v$ and let $B$ be the (unique) $i$-block containing $u$. The response to such an attack is that every guard in $B$ that does not occupy a $\pm i$-face of $B$ moves to its $i$-neighbor in $B$ (if such a neighbor is on the $i$-face of $B$, then the guard will jump to the corresponding vertex in the $-i$-face of $B$ which is possible by Lemma 15). Indeed, any vertex in $B$ that is not on a face of $B$ is occupied by at most $g_{i-1}$ guards, each of the vertices of the $\pm i$-faces of $B$ is occupied by $g_{i}$ guards, and each of the vertices of the $\pm(i+1)$-faces of the $(i+1)$-block $B^{\prime}$ that contains $B$ is occupied by $g_{i+1}$ guards (except if $i=D$, in which case $B^{\prime}=G$, and each of the border vertices of $G$ are occupied by $g_{D}$ guards). Hence, every vertex of the $\pm(i+1)$-faces and of the $\pm i$-faces of 
$B^{\prime}$ is occupied by at least $g_{i}=S_{i}+g_{i-1} S_{i-1}>g_{i-1}$ guards, and thus, Lemma 15 can be applied. Then, since the guards act as if they were in a torus, the level-1 guards maintain the property that they occupy a dominating set of $V(G)$ and so, by induction on the number of attacks, the strategy will protect against any infinite sequence of attacks.

We can now prove Theorem 14 using the strategy described above.

Proof of Theorem 14. We will show that the strategy above can be employed in $G$, and that it only requires $\gamma(G)+o(\gamma(G))$ guards. Thus, we first prove that using $o(\gamma(G))$ guards, we can restrict our study to the case where $n_{D}^{\frac{1}{m_{D}}}$ is an integer, and, for all $1 \leq i \leq D, n_{i} \equiv 0\left(\bmod n_{D}^{\frac{1}{m_{D}}}\right)$, so that we can employ the strategy described in this section. For this purpose, one guard is placed at each of the vertices of a few (in comparison to the size of the entire grid) hyperplanes of $G$ (and these guards will never move) such that what remains to be protected is an $N_{1} \times N_{2} \times \cdots \times N_{D}$ subgrid $H$ such that $N_{D}^{\frac{1}{m_{D}}}$ is an integer and, for all $1 \leq i \leq D, N_{i} \equiv 0\left(\bmod N_{D}^{\frac{1}{m_{D}}}\right)$. Overall, this takes $O\left(n_{1} \ldots n_{D-1} n_{D}^{\frac{m_{D}-1}{m_{D}}}\right)=o(\gamma(G))$ guards, so proving that $\gamma_{\text {all }}^{\infty}(H)=\gamma(H)+o(\gamma(H))$ will be sufficient to prove the theorem. Indeed, for some integer $x>0$, we have that $x \leq n_{D}^{\frac{1}{m_{D}}} \leq x+1$. Therefore, at most $(x+1)^{m_{D}}-x^{m_{D}}=O\left(x^{m_{D}-1}\right) \leq O\left(n_{D}^{\left(\frac{1}{m_{D}}\right)^{m_{D}-1}}\right)=O\left(n_{D}^{\frac{m_{D}-1}{m_{D}}}\right)$ hyperplanes of dimension $n_{1} \times \cdots \times n_{D-1}$ must have one guard placed on each of their vertices so that $N_{D}^{\frac{1}{m_{D}}}$ is an integer, which also ensures that $N_{D} \equiv 0\left(\bmod N_{D}^{\frac{1}{m_{D}}}\right)$. Then, for all $1 \leq i \leq D-1$, to ensure that $N_{i} \equiv 0\left(\bmod N_{D}^{\frac{1}{m_{D}}}\right)$, it suffices to place one guard on each vertex of at most $N_{D}^{\frac{1}{m_{D}}}$ hyperplanes of dimension at most $n_{1} \times \cdots \times n_{D-2} \times N_{D}$. For each $1 \leq i \leq D-1$, this requires at most $O\left(n_{1} \ldots n_{D-2} N_{D}^{\frac{m_{D}+1}{m_{D}}}\right) \leq O\left(n_{1} \ldots n_{D-1} n_{D}^{\frac{1}{m_{D}}}\right.$ ) guards (for a total of $o(\gamma(G)$ ) guards over all $i$ since $D=O(1))$. Hence, we can assume that $n_{D}^{\frac{1}{m_{D}}}$ is an integer, and, for all $1 \leq i \leq D$, $n_{i} \equiv 0\left(\bmod n_{D}^{\frac{1}{m_{D}}}\right)$.

The proof then follows from the fact that the strategy described throughout this section is a winning strategy for the guards in $G$ and, by Lemma 17, it uses $\gamma(G)+o(\gamma(G))$ guards.

\subsection{From $D$-dimensional Cartesian grids to $D$-dimensional strong grids}

To conclude, let us generalise our previous result to a larger graph class. Recall that, for any two graphs $G$ and $H$, the vertex set of the graph $G^{\prime}=G \otimes H$ (strong product of $G$ and $H$ ) is the Cartesian product of the vertex sets of $G$ and $H$, i.e., $V\left(G^{\prime}\right)=V(G) \times V(H)$. For the edges of $G^{\prime}$, distinct vertices $\left(u_{1}, u_{2}\right) \in V\left(G^{\prime}\right)$ and $\left(v_{1}, v_{2}\right) \in V\left(G^{\prime}\right)$ are adjacent if and only if:

- $u_{1}=v_{1}$ and $u_{2}$ is adjacent to $v_{2}$, or

- $u_{2}=v_{2}$ and $u_{1}$ is adjacent to $v_{1}$, or

- $u_{1}$ is adjacent to $v_{1}$ and $u_{2}$ is adjacent to $v_{2}$.

In this section, we consider a large family $\mathcal{F}$ of graphs that are supergraphs of the Cartesian grid $P_{n_{1}} \square \cdots \square P_{n_{D}}$ and subgraphs of the strong grid $P_{n_{1}} \otimes \cdots \otimes P_{n_{D}}$ for any fixed integer $D \geq 2$. 
Informally, $\mathcal{F}$ is the family of supergraphs of the Cartesian grid and subgraphs of the strong grid, where all vertices not on the "border" have the same "symmetric adjacencies".

Definition of the family $\mathcal{F}$. More precisely, the family $\mathcal{F}$ is defined as follows. We start from the Cartesian grid and, for every vertex, we add some of its possible adjacencies in the strong grid. This is done in such a way that, if some adjacency is added, then the "symmetrical" one is also added, and, moreover, all vertices (except on the "border") have similar adjacencies (roughly, the graph is "almost" vertex-transitive).

Let $A^{*}=\left\{\left(a_{1}, \ldots, a_{D}\right) \in\{-1,0,1\}^{D}\left|\sum_{1<i<D}\right| a_{i} \mid>1\right\}$. Let $(A, \bar{A})$ be any fixed partition of $A^{*}$ such that $x \in A$ if and only if $-x \in \bar{A}$ (where $-\left(a_{1}, \ldots, a_{D}\right)=\left(-a_{1}, \ldots,-a_{D}\right)$ ). From now on, let us consider $A$ as an ordered sequence of length $\eta=\frac{3^{D}-1}{2}-D$. A graph $G \in \mathcal{F}$ is any graph obtained as follows.

Let $M \in\{0,1\}^{\eta}$ be any Boolean sequence and let $\mathcal{N}=\left\{n_{1}, \ldots, n_{D}\right\} \subset \mathbb{N}^{* D}$ such that, for all $1 \leq i \leq D-1, n_{i} \geq n_{i+1}$, where $D=O(1)$ is a fixed constant. Furthermore, let $n_{D}=\omega(1)$, so that, for all $1 \leq i \leq D, n_{i}$ is not a fixed constant. Start with $G=G_{D}(M, \mathcal{N})$ equal to the Cartesian grid $P_{n_{1}} \square \cdots \square P_{n_{D}}$. Then, for every $u \in V\left(G_{D}(M, \mathcal{N})\right)$ with coordinates $\left(u_{1}, \cdots, u_{D}\right)$, and for any $j \leq \eta$, let $\left(a_{1}^{j}, \cdots, a_{D}^{j}\right)=A[j]$. Then, the vertex $u^{-}$with coordinates $\left(u_{1}-a_{1}^{j}, \cdots, u_{D}-a_{D}^{j}\right)$ (resp., $u^{+}$with coordinates $\left(u_{1}+a_{1}^{j}, \cdots, u_{D}+a_{D}^{j}\right)$ ) is adjacent to $u$ if and only if $M[j]=1$ and $u^{-}$(resp., $u^{+}$) exists (i.e., if its coordinates $\left(i_{1}, \cdots, i_{D}\right)$ are such that $1 \leq i_{k}=u_{k}-a_{k}^{j} \leq n_{k}$ (resp., $1 \leq i_{k}=u_{k}+a_{k}^{j} \leq n_{k}$ ) for every $1 \leq k \leq D$ (that is, if $u^{-}$(resp., $u^{+}$) is well-defined in $\left.P_{n_{1}} \square \cdots \square P_{n_{D}}\right)$. Two examples of such graphs are given in Figure 15.

Theorem 18. For any graph $G \in \mathcal{F}, \gamma_{\text {all }}^{\infty}(G)=\gamma(G)+o(\gamma(G))$.

Proof. The strategy starts as the one from Theorem 14 for the $D$-dimensional Cartesian grid $G^{\prime}$ from which $G$ is built. Let us assume that an unoccupied vertex $u$ is attacked and let $v \in N(u)$ be its occupied neighbour (exists by induction hypothesis on the number of attacks). If $v$ is a neighbour of $u$ in $G^{\prime}$, then the strategy proceeds as previously in $G^{\prime}$. Otherwise, let $W \subseteq\left\{i_{1}, \ldots, i_{D}\right\}$ be the coordinates in which $u$ differs from $v\left(|W|>1\right.$ since, otherwise, $\left.u v \in E\left(G^{\prime}\right)\right)$. For every $i_{j} \in W$, let $B_{j}$ be the unique $j$-block containing $v$ and $u$. Then, for every $i_{j} \in W$, every guard in $B_{j}$ except those on the $\pm j$-faces of $B_{j}$ (if guards jump, we consider them to be the ones that are moving), moves according to the coordinates $W_{j}$, where $W_{j}=W \backslash\left(\bigcup_{\ell<j} i_{\ell}\right)$. By the previous section (combining moves along $|W|$ coordinates simultaneously), this leads to a new dominating set of $V(G)$ and so, by induction on the number of attacks, the strategy will protect against any infinite sequence of attacks. It is easy to see this is possible in the case where no guards need to move onto a face of a block when moving according to the coordinates $W_{j}$. To see that it works in the case where guards may also need to move onto a face of a block, it suffices to recall that, for all $1 \leq i \leq D$, each vertex of a $\pm i$-face of an $i$-block is occupied by $g_{i}=S_{i}+g_{i-1} S_{i-1}$ guards. Precisely, for all $1 \leq i \leq D$, the $S_{i}$ guards on each vertex of the $\pm i$-faces of each $i$-block allow for Lemma 15 to be applied to guards moving in any $i$-block for a single $i$ at a time. However, for all $2 \leq \ell \leq D+1$, the additional $g_{\ell-1} S_{\ell-1}$ guards on each vertex of the $\pm(\ell)$-faces of each $\ell$-block allow for Lemma 15 to be applied to guards moving in any $(\ell-1)$-block at the same time as it is being applied to guards moving in an $\ell$-block, for all $\ell$ at the same time, as well as all the 1-blocks. 

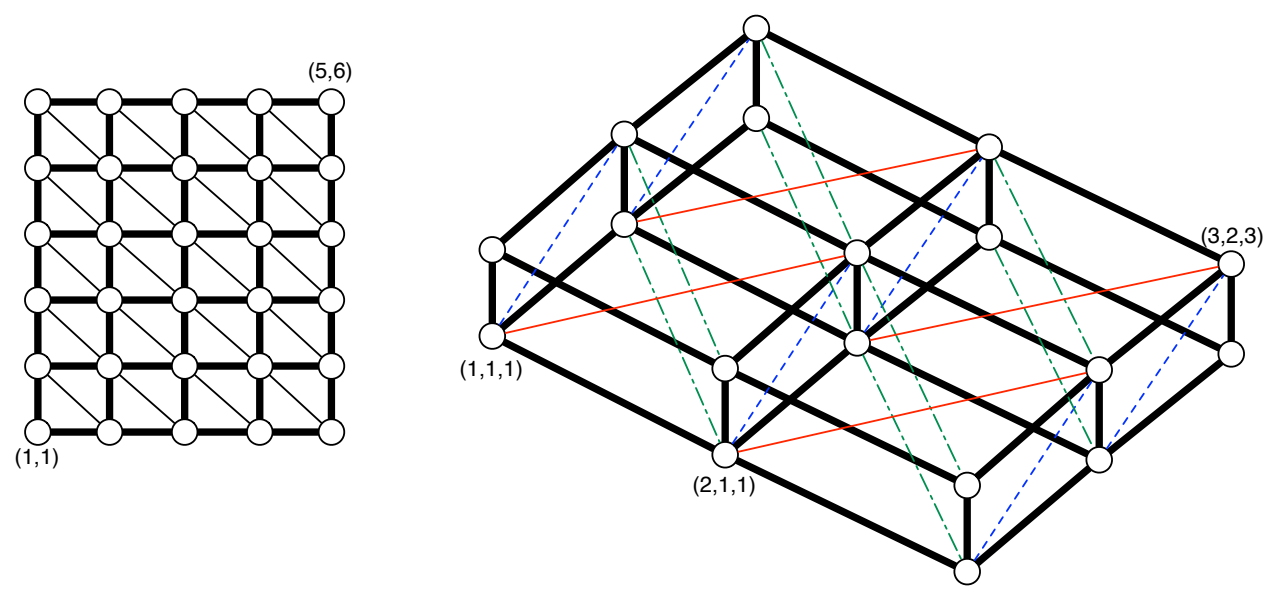

Figure 15: Two examples of graphs in the class $\mathcal{F}$. The edges of the underlying Cartesian grids are in bold. The graph $G_{1}$ on the left is defined as follows: $D=2$ and $A^{*}=\{(-1,-1),(1,-1),(-1,1),(1,1)\}$ (roughly, it represents all adjacencies in the strong grid, not in the Cartesian grid). Then, $A=((-1,1),(1,1))$ (for any two "symmetrical" adjacencies, only one is kept). Finally, the sequence defining $G_{1}$ is $M_{1}=(1,0)$ (meaning that only the first adjacency in $A$ will be taken into account to define $\left.G_{1}\right)$. On the right, the graph $G_{2}$ is defined as follows: $D=3$, $A=\{(-1,1,0),(1,1,0),(-1,-1,1),(0,-1,1),(1,-1,1),(-1,0,1),(1,0,1),(-1,1,1)$, $(0,1,1),(1,1,1)\}$ and $M_{2}=(0,0,0,0,0,1,0,0,1,1)$. The colors of the thin edges correspond to the three classes of adjacencies added to the Cartesian grid to obtain $G_{2}$. 


\section{Further Work}

Our results in the strong grid leave the open problem of tightening the bounds. Concerning the graphs $G \in \mathcal{F}$, where $\mathcal{F}$ is the family of higher dimensional grids defined in Section 6.2, for which we proved that $\gamma_{a l l}^{\infty}(G)=\gamma(G)+o(\gamma(G))$, it would be interesting if a general lower bound of at least $\gamma_{\text {all }}^{\infty}(G)=\gamma(G)+\omega(1)$ could be proven. Moreover, as was shown in Section 3, the upper bound for the eternal domination number of particular grid families can be improved upon by looking at their dominating sets, i.e., we proved that $\gamma_{\text {all }}^{\infty}\left(P_{n} \otimes P_{m}\right)=\gamma\left(P_{n} \otimes P_{m}\right)+O\left(m n^{\frac{1}{2}}\right)$ in Theorem 8, but the strategy used in the proof of Theorem 18 only yields $\gamma_{\text {all }}^{\infty}\left(P_{n} \otimes P_{m}\right)=\gamma\left(P_{n} \otimes P_{m}\right)+O\left(m n^{\frac{2}{3}}\right)$, where $m \geq n$. Thus, another direction for future work is to take a specific type of grid and then use the technique in Section 6 and adapt it to take into account the properties of the dominating sets of that grid, in order to improve the upper bound, as we did for the 2-dimensional strong grid in Section 3. Lastly, as mentioned in the introduction, it is known that given a graph $G$ and an integer $k$ as inputs and asking whether $\gamma_{\text {all }}^{\infty}(G) \leq k$ is NP-hard in general [3] but the exact complexity of the decision problem is open.

\section{References}

[1] J. Arquilla and H. Fredricksen. "graphing" an optimal grand strategy. Military Operations Research, 1(3):3-17, 1995.

[2] G. Bagan, A. Joffard, and H. Kheddouci. Eternal dominating sets on digraphs and orientations of graphs. CoRR, abs/1805.09623, 2018.

[3] S. Bard, C. Duffy, M. Edwards, G. Macgillivray, and F. Yang. Eternal domination in split graphs. J. Comb. Math. Comb. Comput., 101:121-130, 2017.

[4] I. Beaton, S. Finbow, and J.A. MacDonald. Eternal domination numbers of $4 \times n$ grid graphs. J. Comb. Math. Comb. Comput., 85:33-48, 2013.

[5] A. Braga, C. Souza, and O. Lee. The eternal dominating set problem for proper interval graphs. Information Processing Letters, 115, 2015.

[6] A. Burger, E. J. Cockayne, W. R. Gründlingh, C. M. Mynhardt, J. H. van Vuuren, and W. Winterbach. Infinite order domination in graphs. J. Comb. Math. Comb. Comput., 50:179-194, 2004.

[7] N. Cohen, F. Mc Inerney, N. Nisse, and S. Pérennes. Study of a combinatorial game in graphs through linear programming. Algorithmica, 82(2):212-244, 2020.

[8] N. Cohen, N. A. Martins, F. Mc Inerney, N. Nisse, S. Pérennes, and R. Sampaio. Spy-game on graphs: Complexity and simple topologies. Theoretical Computer Science, 725:1-15, 2018.

[9] Reinhard Diestel. Graph Theory, 4th Edition, volume 173 of Graduate texts in mathematics. Springer, 2012.

[10] S. Finbow, M. E. Messinger, and M. F. van Bommel. Eternal domination in $3 \times n$ grids. Australas. J. Combin., 61:156-174, 2015.

[11] A. Gagnon, A. Hassler, J. Huang, A. Krim-Yee, F. Mc Inerney, A. Mejía Zacarías, B. Seamone, and V. Virgile. A method for eternally dominating strong grids. Discrete Mathematics and Theoretical Computer Science, vol. 22 no. 1, 2020.

[12] W. Goddard, S. M. Hedetniemi, and S. T. Hedetniemi. Eternal security in graphs. J. Comb. Math. Comb. Comput., 52:160-180, 2005.

[13] J. L. Goldwasser, W. F. Klostermeyer, and C. M. Mynhardt. Eternal protection in grid graphs. Util. Math., 91:47-64, 2013. 
[14] D. Gonçalves, A. Pinlou, M. Rao, and S. Thomassé. The domination number of grids. SIAM J. Discrete Math., 25(3):1443-1453, 2011.

[15] F. Mc Inerney, N. Nisse, and S. Pérennes. Eternal domination in grids. In Proceedings of the 11th International Conference on Algorithms and Complexity, CIAC 2019, volume 11485 of Lecture Notes in Computer Science, pages 311-322. Springer, Cham, 2019.

[16] W. F. Klostermeyer, M. Lawrence, and G. MacGillivray. Dynamic dominating sets: the eviction model for eternal domination. Manuscript, 2014.

[17] W. F. Klostermeyer and G. MacGillivray. Eternal dominating sets in graphs. J. Comb. Math. Comb. Comput., 68:97-111, 2009.

[18] W. F. Klostermeyer and C. M. Mynhardt. Eternal total domination in graphs. Ars Combin., 68:473-492, 2012.

[19] W. F. Klostermeyer and C. M. Mynhardt. Protecting a graph with mobile guards. Applicable Analysis and Discrete Mathematics, 10, 2014.

[20] I. Lamprou, R. Martin, and S. Schewe. Eternally dominating large grids. Theoretical Computer Science, 794:27-46, 2019.

[21] M. E. Messinger. Closing the gap: Eternal domination on $3 \times n$ grids. Contributions to Discrete Mathematics, 12(1):47-61, 2017.

[22] C. S. Revelle. Can you protect the roman empire? Johns Hopkins Magazine, 50(2), 1997.

[23] C. S. Revelle and K. E. Rosing. Defendens imperium romanum: A classical problem in military strategy. Amer. Math. Monthly, 107:585-594, 2000.

[24] M. Rinemberg and F. J. Soulignac. The eternal dominating set problem for interval graphs. Information Processing Letters, 146:27-29, 2019.

[25] I. Stewart. Defend the roman empire! Scientific American, pages 136-138, 1999.

[26] C. M. van Bommel and M. F. van Bommel. Eternal domination numbers of $5 \times n$ grid graphs. J. Comb. Math. Comb. Comput., 97:83-102, 2016. 